\title{
Tidal features of classical Milky Way satellites in a Lambda cold dark matter universe
}

\section{Wang, M. -Y.}

2017-07

Wang , M -Y , Fattahi , A , Cooper , A P , Sawala , T , Strigari , L E , Frenk , C S , Navarro , J F , Oman , K \& Schaller , M 2017, ' Tidal features of classical Milky Way satellites in a Lambda cold dark matter universe ' , Monthly Notices of the Royal Astronomical Society , vol. 468 , no. 4 , pp. 4887-4901 . https://doi.org/10.1093/mnras/stx742

http://hdl.handle.net/10138/209211

https://doi.org/10.1093/mnras/stx742

unspecified

publishedVersion

Downloaded from Helda, University of Helsinki institutional repository.

This is an electronic reprint of the original article.

This reprint may differ from the original in pagination and typographic detail.

Please cite the original version. 


\title{
Tidal features of classical Milky Way satellites in a $\Lambda$ cold dark matter universe
}

\author{
M.-Y. Wang, ${ }^{1 \star}$ Azadeh Fattahi, ${ }^{2}$ Andrew P. Cooper, ${ }^{3}$ Till Sawala, ${ }^{4}$ Louis E. Strigari, ${ }^{1}$ \\ Carlos S. Frenk, ${ }^{3}$ Julio F. Navarro, ${ }^{2}$ Kyle Oman ${ }^{2}$ and Matthieu Schaller ${ }^{3}$ \\ ${ }^{1}$ Department of Physics and Astronomy, Mitchell Institute for Fundamental Physics and Astronomy, Texas A\&M University, \\ College Station, TX 77843-4242, USA \\ ${ }^{2}$ Department of Physics and Astronomy, University of Victoria, PO Box 1700 STN CSC, Victoria, BC V8W 2Y2, Canada \\ ${ }^{3}$ Institute for Computational Cosmology, Department of Physics, University of Durham, South Road, Durham DH1 3LE, UK \\ ${ }^{4}$ Department of Physics, University of Helsinki, Gustaf Hallstromin katu 2a, FI-00014 Helsinki, Finland
}

Accepted 2017 March 23. Received 2017 March 13; in original form 2016 November 17

\begin{abstract}
ABSTRA CT
We use the APOSTLE (A Project Of Simulating The Local Environment) cosmological hydrodynamic simulations to examine the effects of tidal stripping on cold dark matter subhaloes that host three of the most luminous Milky Way dwarf satellite galaxies: Fornax, Sculptor and Leo I. We identify simulated satellites that match the observed spatial and kinematic distributions of stars in these galaxies, and track their evolution after infall. We find $\sim 30$ per cent of subhaloes hosting satellites with present-day stellar mass $10^{6}-10^{8} \mathrm{M}_{\odot}$ experience $>20$ per cent stellar mass-loss after infall. Fornax analogues have earlier infall times compared to Sculptor and Leo I analogues. Star formation in Fornax analogues continues for 3-6 Gyr after infall, whereas Sculptor and Leo I analogues stop forming stars $<2-3$ Gyr after infall. Fornax analogues typically show more significant stellar mass-loss and exhibit stellar tidal tails, whereas Sculptor and Leo I analogues, which are more deeply embedded in their host dark matter haloes at infall, do not show substantial mass-loss due to tides. When additionally comparing the orbital motion of the host subaloes to the measured proper motion of Fornax, we find the matching more difficult; host subhaloes tend to have pericentres smaller than that measured for Fornax itself. From the kinematic and orbital data, we estimate that Fornax has lost 10-20 percent of its infall stellar mass. Our best estimate for the surface brightness of a stellar tidal stream associated with Fornax is $\Sigma \sim 32.6 \mathrm{mag} \operatorname{arcsec}^{-2}$, which may be detectable with deep imaging surveys such as DES and LSST.
\end{abstract}

Key words: methods: numerical-Galaxy: evolution-Galaxy: kinematics and dynamicsLocal Group-dark matter.

\section{INTRODUCTION}

Milky Way (MW) dwarf spheroidal (dSph) satellite galaxies are among the most dark-matter- (DM) dominated stellar systems in the Universe (Mateo 1998; McConnachie 2012; Walker 2013). High mass-to-light ratios in these systems have been derived from their velocity dispersion under the assumption that their stellar populations are in dynamical equilibrium. However, this assumption of dynamical equilibrium has been called into question, and must continue to be scrutinized as larger kinematic samples and deeper photometric surveys become available.

Around the MW, in addition to the clear case of Sagittarius (Ibata, Gilmore \& Irwin 1994), among the 'classical' dSphs, only Carina (Muñoz et al. 2006; Battaglia et al. 2012; McMonigal et al. 2014) and a few 'ultra-faint' dwarf galaxies (e.g. the Hercules dSph; Roderick et al. 2015) show signs of having been affected by tides. Around M31, there is evidence of ongoing tidal disturbance in several satellite galaxies (Choi, Guhathakurta \& Johnston 2002; Crnojević et al. 2014), and similar findings have been made in a study of NGC 205 (Geha et al. 2006). Although tidal features are not evident in the majority of $\mathrm{dSphs}$, this may be simply because photometric data sets are not sufficiently deep and wide enough to identify them.

Models of galaxy formation in a $\Lambda$ cold dark matter (CDM) cosmogony can only reproduce the present-day properties of the MW satellite system if a fraction of satellites experience significant mass-loss as the result of tidal interactions with their host ( $\mathrm{Li}, \mathrm{De}$ Lucia \& Helmi 2010; Barber et al. 2015; Sawala et al. 2016). Tidal stirring may also be an effective mechanism for transforming discy field dwarfs into objects with properties resembling dSphs (Mayer et al. 2001; Tomozeiu, Mayer \& Quinn 2016). Tidal stripping of 
satellite galaxies can also provide a unique test of the nature of DM. For example, satellite galaxies are more vulnerable to tides if their gravitational potentials are shallow or cored (Errani, Peñarrubia \& Tormen 2015), for example, due to DM self-interactions (Dooley et al. 2016). Different DM scenarios, for example, warm DM (WDM; Lovell et al. 2014) or late-decaying DM (DDM; Wang et al. 2014), also predict different subhalo assembly histories for a given dSph. Wang et al. (2016) show that the CDM subhaloes that best match the stellar mass and kinematics of Fornax are those that have been severely tidally stripped.

Several previous authors have used $N$-body simulations to examine the observational signatures of tidal interactions on MW satellite galaxies. Muñoz, Majewski \& Johnston (2008) examine whether the observed features of the Carina dSph can be accounted for by a mass-follows-light model that includes tidal disruption, finding that tidal stripping can strongly affect stars at large radii. Peñarrubia, Navarro \& McConnachie (2008) find that once stars start to be stripped from a dSph, the dark-to-luminous mass ratio increases, and they consider the implications of this for the known dSphs. Battaglia, Sollima \& Nipoti (2015) simulate subhaloes with orbits and structural properties that match the Fornax dSph, and find that the structure and kinematics of the stars are only mildly affected by tidal interactions. Klimentowski et al. (2007) study how unbound particles affect the interpretation of stellar kinematics, with a specific application to Fornax. Read et al. (2006) also examine the effects of tidal stripping and tidal shocking on the dSph velocity dispersion profiles.

In this paper, using high-resolution (HR) cosmological hydrodynamical simulations from the APOSTLE (A Project Of Simulating The Local Environment) project, we match simulated satellites, and hence subhaloes, to the observable properties of bright MW dSphs. We examine the nature of faint stellar tidal features associated with these subhaloes and make predictions applicable to real dSphs. Our study is the first attempt to examine the formation of dSph tidal features in an cosmological context with full hydrodynamical models. We specifically focus on satellites in the stellar mass range $M_{*}=10^{6}-10^{8} \mathrm{M}_{\odot}$, which are very well resolved by the APOSTLE simulations; the classical MW dSphs that fall within this mass range are Fornax, Sculptor and Leo I. We predict the surface brightness of tidal streams associated with simulated dSphs, and compare to the surface brightness of known diffuse stellar substructures in the halo of the MW. We also consider the possibility that unbound stars contaminate existing kinematic data sets for these galaxies.

The outline of this paper is as follows. In Section 2, we briefly describe the simulations used in our analysis. In Section 3, we review our procedure for constructing a subhalo sample from the simulations by matching to observational data. In Section 4, we show the general effects of gravitational tides on subhaloes, including the effects on DM and stellar mass-loss, and changes to the galaxy stellar velocity dispersion. In Sections 5 and 6, we present our results, including the overall properties and mass assembly histories of the bright $\mathrm{dSph}$ analogues and the detectability of tidal features around them. Finally, we summarize our conclusions in Section 7.

\section{SIMULATIONS}

We use sets of HR Local Group (LG) simulations from the APOSTLE project. For details, we refer the readers to the original simulation papers (Fattahi et al. 2016a; Sawala et al. 2016). Here we briefly describe some basic properties of the simulations.

The simulations were performed with the code developed for the EAGLE (Evolution and Assembly of GaLaxies and their Environ- ments) project (Crain et al. 2015; Schaye et al. 2015). The EAGLE code is a modified version of P-GADGET-3, which is an improved version of the publicly available GADGET- 2 code (Springel 2005). The EAGLE model has been calibrated to reproduce the $z=0.1$ stellar mass function and galaxy sizes from $10^{8}$ to $10^{11} \mathrm{M}_{\odot}$, and shown to successfully reproduce many properties and scaling laws of observed galaxy populations, such as the evolution of the stellar mass function (Furlong et al. 2015), and the luminosities and colours of galaxies (Trayford et al. 2015). For our study, we utilize both the medium-resolution (MR) and HR runs of the APOSTLE simulations (corresponding to L2 and L1 resolutions in Sawala et al. 2016). The larger number of MR runs allows us to explore a more representative sample of satellite orbits and hence gravitational tidal effects, whereas the HR runs reveal more details of the internal structure of satellite galaxies and their stellar tidal features. The gas, stellar and DM components of satellites are shaped by the galaxy formation process and environmental effects after infall. Examples of satellite properties in the simulations can be found in Sawala et al. (2016). Due to changes to the structure of haloes and the evolution in the LG environment, the simulations well reproduce the observed relation between stellar mass and velocity dispersion of dSph galaxies (Fattahi et al. 2016b; Sawala et al. 2016). The HR runs consist of three LG realizations (AP-1, AP-4 and AP-11 in Fattahi et al. 2016a), which contain six MW-mass host haloes with $M_{200}=1.66 \times 10^{12}, 1.10 \times 10^{12}, 1.38 \times 10^{12}, 1.35 \times 10^{12}$, $0.99 \times 10^{12}$ and $0.80 \times 10^{12} \mathrm{M}_{\odot}$ at $z=0 . M_{200}$ is defined as the halo mass enclosed within a density contour 200 times greater than the critical density of the Universe. The primordial gas particle mass of AP-1 (AP-4, AP-11) is $0.99(0.49,0.84) \times 10^{4} \mathrm{M}_{\odot}$; the $\mathrm{DM}$ particle mass is $5.92(2.92,5.02) \times 10^{4} \mathrm{M}_{\odot}$. The maximum gravitational softening length is $134 \mathrm{pc}$ for all three HR runs.

We also include the $12 \mathrm{LG}$ pairs of MR runs in our analysis. The total mass of each pair (i.e. the sum of the virial masses of the two primary haloes) is in the range $\log _{10} M_{\text {tot }} / \mathrm{M}_{\odot}=[12.2,12.6]$. The primordial gas particle mass spans the range $(1.09-1.27) \times 10^{5} \mathrm{M}_{\odot}$, and the DM particle mass range is $(6.52-7.59) \times 10^{5} \mathrm{M}_{\odot}$. The maximum gravitational softening length is $307 \mathrm{pc}$ for all MR runs. The APOSTLE simulations were performed in the WMAP7 cosmology (Komatsu et al. 2011), with density parameters at $z=0$ for matter, baryons and dark energy of $\Omega_{\mathrm{m}}=0.272$, $\Omega_{\mathrm{b}}=0.0455$ and $\Omega_{\Lambda}=0.728$, respectively, Hubble parameter $H_{0}=70.4 \mathrm{~km} \mathrm{~s}^{-1} \mathrm{Mpc}^{-1}$, linear power spectrum amplitude on the scale of $8 h^{-1} \mathrm{Mpc} \sigma_{8}=0.81$ and power-law spectral index $n_{\mathrm{s}}=0.967$. DM haloes in both simulations were identified using the friends-of-friends (FoF) algorithm. Each FoF halo was iteratively decomposed into self-bound subhaloes using the SUBFIND algorithm (Springel et al. 2001).

\section{METHODOLOGY}

In this section, we outline how we identify subhaloes in APOSTLE simulations that provide good matches to the observed stellar kinematics of particular dSphs. We review the procedure outlined in Wang et al. (2016), based on the Jeans formalism, which we follow closely here, and also the dynamical mass estimation in Fattahi et al. (2016b), which we adopt for selecting MR analogues. We then describe how we identify tidally stripped stars previously associated with simulated dSphs.

\subsection{Selecting simulated analogues of real dSphs}

We focus on MW satellites in the stellar mass range $10^{6} \leq M_{\star} \leq$ $10^{8} \mathrm{M}_{\odot}$. Three of the so-called classical MW dSph satellite galaxies 
have a stellar mass in this range (assuming a stellar mass-to-light ratio of order unity): Fornax (absolute magnitude $M_{V}=-13.2$ ), Sculptor $\left(M_{V}=-11.1\right)$ and Leo I $\left(M_{V}=-11.9\right)$ (Mateo 1998). These three dSphs have similar line-of-sight (LOS) stellar velocity dispersion $\left\langle\sigma_{*}\right\rangle \sim 9-11 \mathrm{~km} \mathrm{~s}^{-1}$ (e.g. Koch et al. 2007 on Leo I, Battaglia et al. 2008 on Sculptor, Battaglia et al. 2006 on Fornax, and also see Mateo, Olszewski \& Walker 2008; Walker, Mateo \& Olszewski 2009).

We consider a sequence of increasingly stringent conditions to select potential bright $\mathrm{dSph}$ analogues in the simulations, as follows:

(i) Select subhaloes with stellar masses broadly consistent with the observed dSph luminosity, assuming a stellar mass-to-light ratio $M / L=0.5-2.0$. We choose the acceptable $z=0$ Fornax stellar mass range to be $(0.6-6.0) \times 10^{7} \mathrm{M}_{\odot}$. For both Sculptor and Leo $\mathrm{I}$, the corresponding range is $(1.0-6.0) \times 10^{6} \mathrm{M}_{\odot}$. Observational estimates of stellar mass are $\sim(1.7-4.3) \times 10^{7} \mathrm{M}_{\odot}$ for Fornax, $\sim 0.2 \times 10^{7} \mathrm{M}_{\odot}$ for Sculptor and $\sim 0.5 \times 10^{7} \mathrm{M}_{\odot}$ for Leo I (Wolf et al. 2010; de Boer et al. 2012; McConnachie 2012).

(ii) Apply the Jeans formalism to select HR subhaloes with gravitational potentials that match the observed stellar kinematics, using the methods described in Section 3.2. The spatial resolution of the MR simulations is not high enough to resolve the subhalo potential within the dSph half-light radius, so instead we use $V_{1 / 2}$ (the circular velocity at the half-light radius), as described in Fattahi et al. (2016b), to constrain the stellar kinematic properties of our dSphs in these simulations.

(iii) Determine how well the orbits of these subhaloes match those derived from the observed mean radial velocity and proper motion of the real dSphs by selecting those analogues at least with comparable orbital pericentres.

\subsection{Fitting dSph stellar kinematics}

To identify subhaloes in the HR simulations with observed stellar kinematic and photometric data to match Fornax, Sculptor and Leo I, we use the spherical Jeans equations, allowing for a constant but non-zero anisotropy in the stellar velocity dispersion. We follow a similar approach to that outlined in Wang et al. (2016), to which we refer the reader for more details.

We begin by identifying subhaloes in the simulations with the halo finder SUBFIND. For each subhalo, the self-bound DM distribution is determined as a function of radius. Given the potential of each subhalo, the parametrized stellar distribution and velocity anisotropy, we solve the spherical Jeans equations to determine the LOS velocity dispersion assuming that the potential is spherically symmetric, dispersion-supported and in dynamical equilibrium. The derived velocity dispersion is then fitted to the stellar kinematic data by marginalizing over model parameters via a Markov chain Monte Carlo method to determine the best-fitting values.

The dSph stellar kinematic data we use are LOS velocity measurements from the samples described in Walker et al. (2009). In this sample, we consider only stars with $>90$ per cent probability of membership. We bin the velocity data in circular annuli and derive the mean LOS velocity in each annulus as a function of projected radius. The LOS velocity dispersion and associated error in each annulus are calculated following the method described in Strigari, Frenk \& White (2010).

Since MR subhaloes have poor resolution of the potential within the half-light radius of the dSphs we study, we adopt the approach described in Fattahi et al. (2016b) to identity the kinematic properties of these subhaloes. We select MR subhaloes that match the observed value of $V_{1 / 2}=\sqrt{G M_{1 / 2} / r_{1 / 2}}$, which is the circular velocity at the half-light radius $r_{1 / 2}$ with estimated enclosed mass $M_{1 / 2}$. The values obtained for each MW classical dSph are listed in Fattahi et al. (2016b). The radii where $V_{1 / 2}$ is measured in the MR runs are smaller than the convergence radius defined by Power et al. (2003). To correct for this numerical resolution, we use the approach described in appendix A of Fattahi et al. (2016b). For example, to find Fornax analogues, we select central ${ }^{1}$ galaxies in the stellar mass range of interest and measure the level of correction for their $V_{1 / 2}$. We then apply an average correction to $V_{1 / 2}$ of satellite galaxies in the same stellar mass range.

\subsection{Tracing stellar tidal features}

An important part of our analysis is to determine the mass of DM and stars lost by a given dwarf satellite galaxy after it falls into its Galactic-analogue host halo and the corresponding evolution of stellar tidal features. To do this, we identify each dwarf galaxy at its time of infall (using a merger tree algorithm that links haloes and subhaloes between simulation outputs). We then consider the set of all stellar, gaseous and DM particles that were gravitationally bound to this galaxy at any time after the infall time. We trace all these particles down to $z=0$. If any new particles are added to the system by ongoing star formation or the gravitational capture of smaller systems between the infall time and the present day, these will not be included in our procedure. It is then trivial to calculate, for example, the fraction of stellar mass lost since infall, and to separate particles at any time into those that have been stripped and those that remain bound to the satellite.

\section{EFFECTS OF TIDES ON SUBHALOES}

In the upper panel of Fig. 1, we plot the fraction of stellar mass that has been lost since infall for all luminous $\left(10^{6} \leq M_{\star} \leq 10^{8} \mathrm{M}_{\odot}\right)$ subhaloes. This fraction is computed using only those star particles that are bound to the subhalo at the time of infall and therefore does not include any stars formed (or accreted) after infall. This additional stellar mass is, however, included in the present-day stellar mass plotted on the $y$-axis of Fig. 1. We use different colours to highlight HR and MR analogues of MW dSphs that we identify on the basis of their stellar kinematics (dark blue for HR Fornax, light blue for MR Fornax and green for HR Sculptor/Leo I analogues). The rest of the subhaloes are shown as grey circles (dark grey for HR and light grey for MR). We will describe the properties of these dSph analogues in detail in Section 5.

In general, we find that present-day subhaloes with stellar mass in the range we study have experienced very different degrees of stellar mass-loss. This reflects the diversity of their orbital properties and DM halo masses and sizes. We find that 36 out of 127 ( 28 per cent) subhaloes (taking HR and MR together) in the present-day stellar mass range shown have lost $\geq 20$ per cent of the stellar mass they had at infall. Among the subhaloes we identify as bright dSph analogues (of both Fornax and Sculptor/Leo), 11 out of 39 ( $\sim 28$ per cent) have lost more than 20 per cent, and majority of them are Fornax analogues. Some of these subhaloes have undergone even more significant stellar stripping, with mass-loss $\geq 40$ per cent for $\sim 19$ per cent of all subhaloes and $\sim 23$ per cent of dSph analogues. Notably, 10 out of 21 Fornax analogues (both HR and MR) have stellar mass-loss fractions $\geq 20$ per cent. On the other

\footnotetext{
${ }^{1}$ Central refers to the most massive structure of a given FoF group.
} 

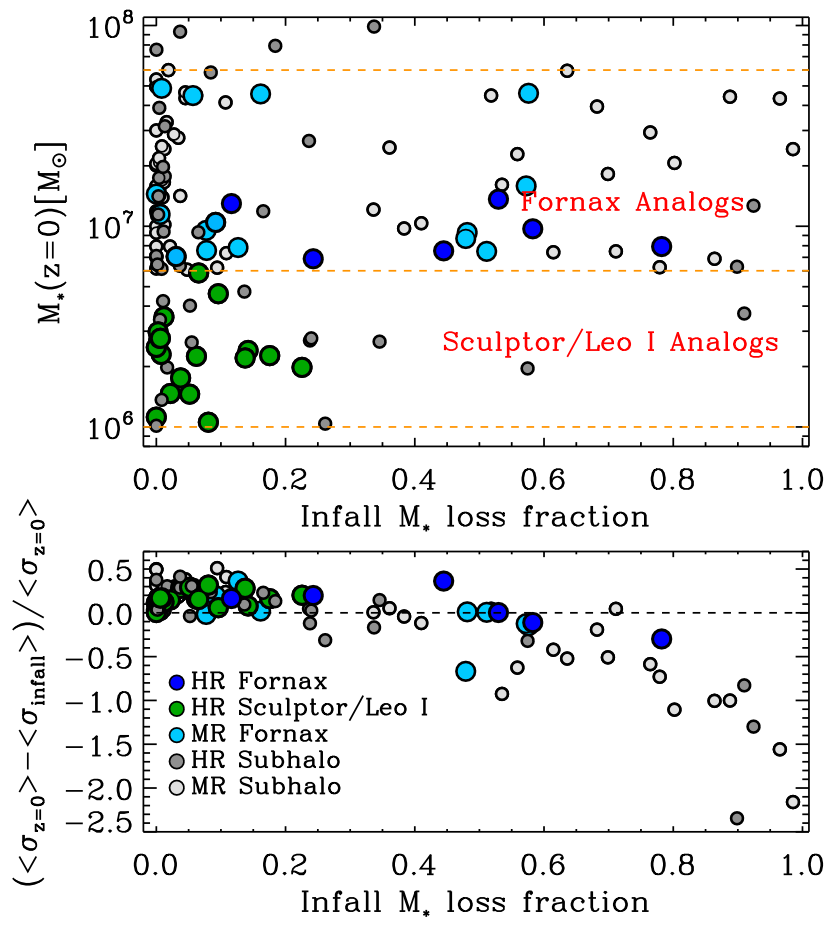

Figure 1. Upper panel: fraction of stellar mass lost since infall for all luminous subhaloes with stellar mass in the range $10^{6} \leq M_{*} \leq 10^{8} \mathrm{M}_{\odot}$ at $z=0$. The $y$-axis gives the stellar mass at $z=0$, which may not equal the infall stellar mass due to mass-loss and star formation after infall. Lower panel: fractional difference between the stellar velocity dispersion at infall and at present time as a function of stellar mass-loss. The dark blue points represent HR Fornax analogues, and the light blue points represent MR Fornax analogues. The green points represent HR Sculptor/Leo analogues. Dark (light) grey points show the rest of the subhaloes from HR (MR) simulations.

hand, all Sculptor/Leo I analogues have a mild stellar mass-loss, $\lesssim 20$ per cent. This result agrees with other studies of tidal effects on MW dSphs using the APOSTLE simulations (Sawala et al. 2016; Fattahi et al. 2016b). These studies show that present-day haloes that have $V_{\max }<30 \mathrm{~km} \mathrm{~s}^{-1}$ and host observable dwarf galaxies have been affected by tidal stripping even more strongly than typical satellite haloes of the same mass. Similar findings are also shown in Sawala et al. (2016); in particular, dwarf galaxies in APOSTLE simulations are biased towards stronger stripping compared to haloes that did not form stars. As pointed out in Sawala et al. (2016), this is an important factor in explaining why these simulations do not demonstrate the 'too big to fail' problem posed by Boylan-Kolchin, Bullock \& Kaplinghat (2011). Fattahi et al. (2016b) also show that tidal stripping may have significantly reduced the DM content of several MW dSphs. Fornax is a clear case for which the value of $\mathrm{V}_{1 / 2}$ is systematically lower than that expected for satellites with comparable luminosity (also see Cooper et al. 2010; Guo et al. 2015 for similar arguments).

In the lower panel of Fig. 1, we show the fractional change in stellar velocity dispersion between the infall time and the present day as a function of the fraction of total mass lost since infall. We can interpret these results in the context of previous studies that showed how tidal forces and mass-loss affect the stellar velocity dispersion of dwarf satellite galaxies (e.g. Peñarrubia et al. 2008). For modest amounts of total mass-loss ( $\leq 20$ per cent), we find an increase in stellar velocity dispersion by up to 50 per cent. This is likely due to a combination of tidal heating, ongoing star formation and (in a small number of cases) ongoing accretion of stars from bound companions. For subhaloes that lose $\geq 90$ per cent of their total mass after infall, the stellar velocity dispersion drops drastically. In intermediate cases (total mass-loss fractions of 20-70 per cent), the change in stellar velocity dispersion follows a smooth evolution between those two regimes with some scatters, reflecting the changing balance between the different effects.

It is expected that the effects of tides on stellar components are highly correlated with the degree to which the DM halo is tidally stripped. The galaxies are embedded deeply inside the DM potential wells, thus subhaloes will typically experience significant DM halo mass-loss before the main stellar distribution begins to show signs of tidal disturbance. In Fig. 2, we show the infall stellar mass-loss fraction as a function of the ratio of subhalo circular velocity at the present day to that at infall, $V_{\max }(z=0) / V_{\max }\left(z_{\text {infall }}\right)$, for subhaloes (both HR and MR) with stellar mass in the range $6 \times 10^{6} \leq M_{*} \leq$ $1 \times 10^{8} \mathrm{M}_{\odot}$. We verify that the tidal effects on the DM and stellar components of subhaloes are consistent with this picture: There is no significant stellar tidal stripping (e.g. $\gtrsim 10$ per cent) until the DM halo is severely stripped [e.g. $V_{\max }(z=0) / V_{\max }\left(z_{\text {infall }}\right) \lesssim$ 70 percent]. We find that the relation between infall stellar massloss fraction $\left(f_{\star}\right.$, loss $)$ and change in $V_{\max }$ is well represented by the following fitting function:

$f_{*, \text { loss }}=\exp \left(-\gamma x^{\alpha}\right)$,

where $x=V_{\max }(z=0) / V_{\max }\left(z_{\text {infall }}\right)$. The best-fitting values of the parameters are $\gamma=13.32$ and $\alpha=3.83$. The fit is shown by the red dashed line in Fig. 2.

\section{PROPERTIES OF DSPH ANALOGUES}

In this section, we discuss the properties of the simulated bright $\mathrm{dSph}$ analogues that are most likely to be determined by their tidal stripping, including their present-day densities and velocity profiles, and the evolution of their stellar, gaseous and dark mass before and after infall. We also discuss about their orbital motions, which indicate the tidal stripping process, and compare to those estimated for their observed counterparts.

\subsection{Density and velocity profiles}

In the upper panels of Fig. 3, we show the circular velocity profiles of $z=0$ subhaloes in the HR runs that match the observed surface density of giant stars in Fornax, Sculptor and Leo I and corresponding radial velocity measurements. Here, only these internal properties are used as constraints. The estimates of circular velocity at the half-light radius $\left(V_{1 / 2}\right)$ for each dSph (from Fattahi et al. 2016b, marked by black diamonds with uncertainties of 10-90th percentile intervals) agree well with the results of our full Jeans analysis. The projected stellar density profiles also agree well with observations (shown in the lower panels of Fig. 3 as black diamonds with Poisson errors). The observed star count (number density) profiles are taken from Coleman et al. (2005) (Fornax, lower left-hand panel), Battaglia et al. (2008) (Sculptor, lower middle panel) and Smolčić et al. (2007) (Leo I, lower right-hand panel). We have scaled the stellar mass density profiles from the simulations to match the amplitude of the observed number density profiles of bright giants. For the simulated Fornax circular velocity curves, the maximum circular velocity $V_{\max }$ at $z=0$ is in the range $17-20 \mathrm{~km} \mathrm{~s}^{-1}$. In contrast to Fornax, the Sculptor and Leo I analogues have a wider $V_{\max }$ range with values up to $28 \mathrm{~km} \mathrm{~s}^{-1}$. These $V_{\max }$ ranges agree well with previous works (e.g. Peñarrubia et al. 2008; Strigari et al. 2010; 


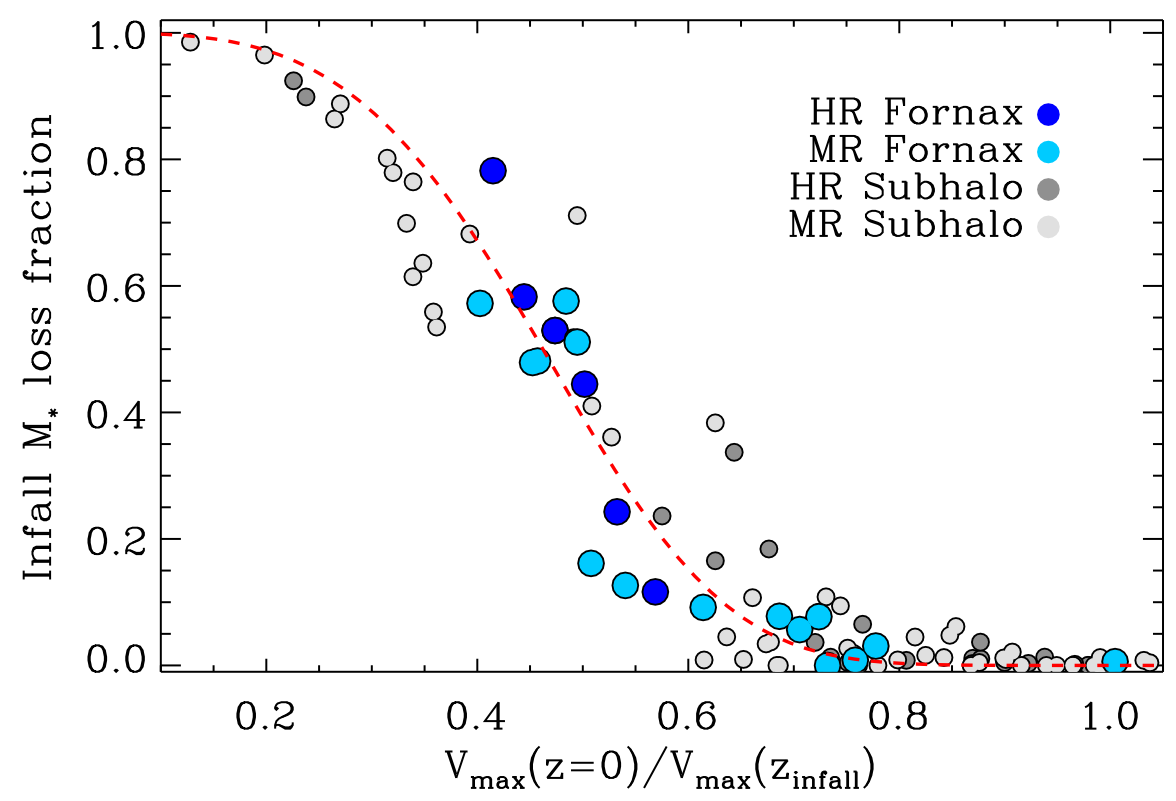

Figure 2. Fraction of stellar mass lost since infall as a function of $V_{\max }(z=0) / V_{\max }\left(z_{\text {infall }}\right)$ for subhaloes with stellar mass in the range $6 \times 10^{6} \leq M_{*} \leq$ $10^{8} \mathrm{M}_{\odot}$ at $z=0$. The dark blue points represent the HR Fornax analogues, and light blue points represent MR Fornax analogues. The dark (light) grey points show the rest of the subhaloes from the HR (MR) simulations in this stellar mass range. The red dashed line illustrates the result of our fitting of equation (1) to these data.
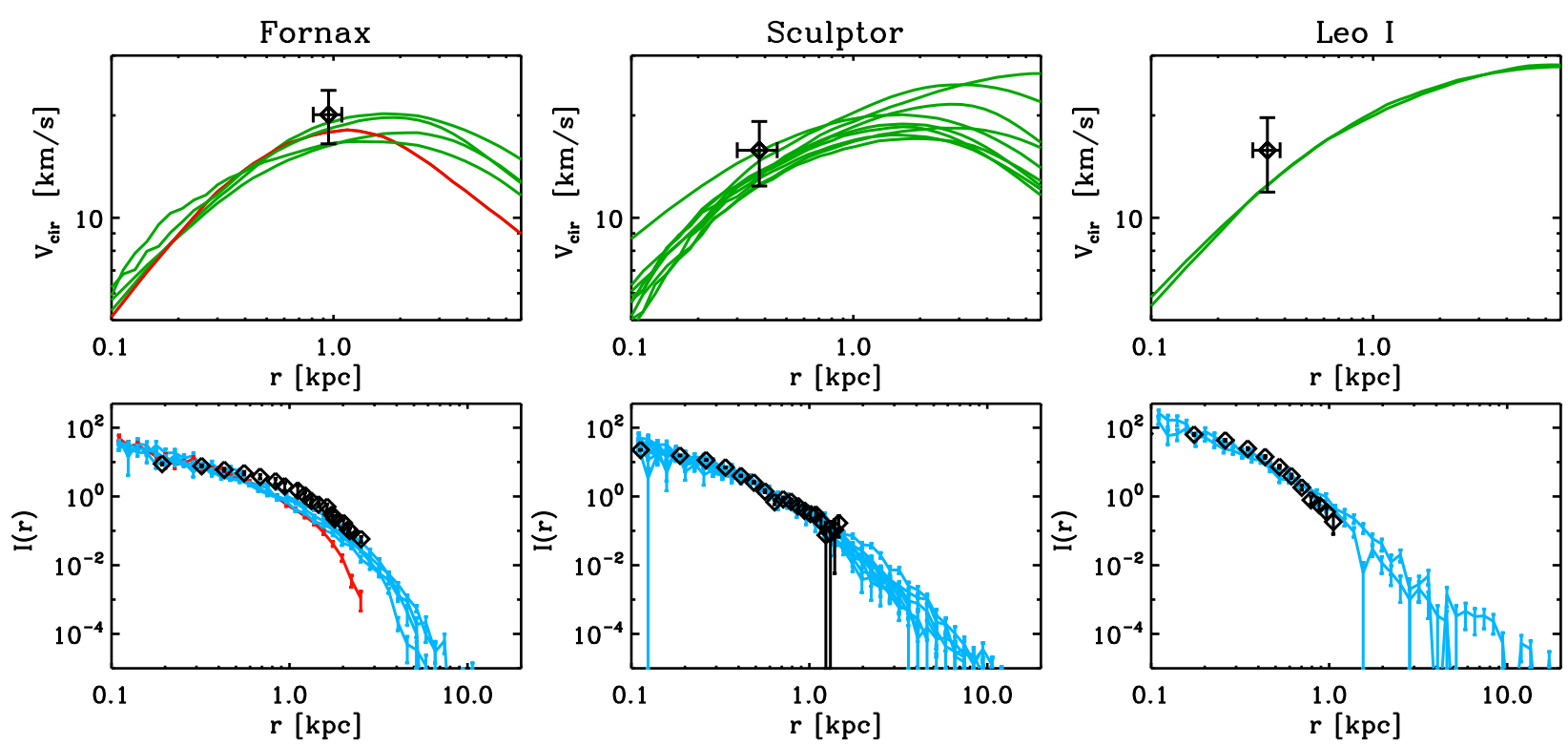

Figure 3. Circular velocity curves (upper panels) and stellar surface density profiles (lower panels) for those subhaloes that match the Fornax (left-hand panel), Sculptor (middle panel) and Leo I (right-hand panel) data at $z=0$. The derived $V_{1 / 2}$ with uncertainties of 10-90th percentile intervals for each dSph from Fattahi et al. (2016b) is marked by a black diamond in the upper panels. The photometric data (black diamonds) in the lower panels are from Coleman et al. (2005) (Fornax, lower left-hand panel), Battaglia et al. (2008) (Sculptor, lower middle panel) and Smolčić et al. (2007) (Leo I, lower right-hand panel). The normalization of the projected stellar density profiles is arbitrary, and the profiles from simulations are projected along an arbitrary direction. The red lines in the Fornax panels highlight the subhalo that suffers the greatest amount of stellar mass-loss ( 78 per cent) among the analogues we identify (see Fig. 1).

Boylan-Kolchin, Bullock \& Kaplinghat 2012; Fattahi et al. 2016b; Sawala et al. 2016), even though some of those analyses were carried out using DM-only simulations or assuming NFW potentials. The Fornax stellar density profiles steepen beyond $r>1 \mathrm{kpc}$, whereas Sculptor and Leo I have somewhat shallower profiles. We find that the average stellar velocity dispersion of the bound star particles is $\sim 9-11 \mathrm{~km} \mathrm{~s}^{-1}$, which agrees well with the observed values. This indicates that those systems are in equilibrium, and hence confirms that our use of the Jeans formalism is appropriate for the simulated systems. We note that Sculptor and Leo I have very similar stellar kinematic properties and light profile shapes. The sets of Sculptor and Leo I analogues have many subhaloes in common. Therefore, we do not distinguish between them in the rest of this paper, except in the discussion of their orbital properties.

In the leftmost panels of Fig. 3, we highlight in red the circular velocity and density profile of one particular Fornax analogue. The density profile of this analogue is significantly shallower than those of the other analogues and is a relatively poor fit to the observed 

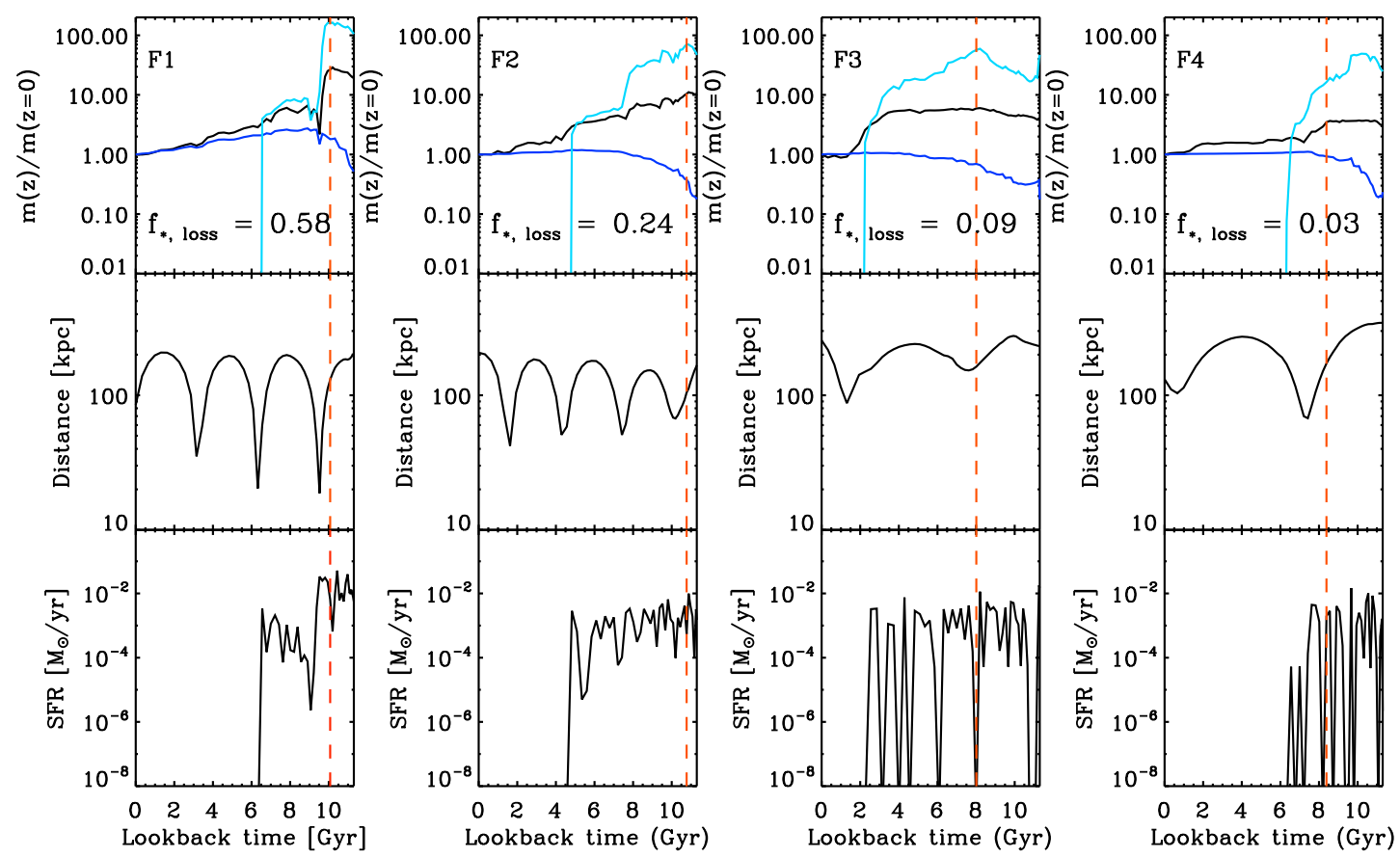

Figure 4. Mass assembly histories (upper row), distance to the host MW halo centre (middle row) and star formation rates for four Fornax analogues as a function of time. Each column shows the properties of one analogue (F1-F4). In the upper row, the black solid lines mark the redshift evolution of the subhalo total mass, dark blue lines are for the stellar component and cyan lines are for the gas mass. The gas mass is normalized to the stellar mass at $z=0$. The red dashed lines indicate the subhalo infall time to their host haloes. The infall stellar mass-loss fractions $\left(f_{\star}\right.$, loss $)$ are labelled for each analogue. The analogues shown here are chosen to have different value of $f \star$, loss (high to low from the left- to right-hand side).

Fornax light profile. This analogue also has a smaller value of $r_{\max }$ (the radius corresponding to $V_{\max }$ ) than the other Fornax analogues. These features indicate that this subhalo has experienced a very significant amount of tidal stripping; we show in the next section that it has lost $\sim 78$ per cent of its stellar mass at infall. As noted by Peñarrubia et al. (2008), even though stars deeply embedded within DM haloes are quite resilient to disruption, the loss of a significant fraction of their total mass, nevertheless, leads to an increase in the concentration of their light profiles. This seems to be the case for our analogue.

\subsection{Mass evolution}

In the upper panels of Fig. 4, we show the evolution of total mass (black lines), stellar mass (dark blue lines) and gas mass (light blue lines) for four Fornax analogues, measured relative to their values at $z=0$. (The gas mass is normalized to the stellar mass at $z=0$.) These are computed by summing the masses of all particles gravitationally bound to the particular halo at each instant according to the SUBFIND algorithm. $^{2}$

We find that the subhaloes of many Fornax analogues show large differences, typically an order of magnitude, between their mass at

\footnotetext{
${ }^{2}$ The assignment of particles to subhaloes in this algorithm is sensitive to the density contrast and velocity of the subhaloes relative to their host halo. Some material (in extreme cases, the whole subhalo) may be considered unbound near pericentre but later considered bound again as the subhalo moves back towards apocentre. This gives rise to transient 'dips' in the bound mass near apocentres, which are visible in Figs 4 and 5. This feature of the SUBFIND algorithm does not affect our discussion here. In most cases, the extent of the subhalo according to the definition used by SUBFIND is a good approximation to the true subhalo tidal radius.
}

$z=0$ and the maximum total mass they reach over their lifetime. That peak mass occurs around the time of infall, which, for these objects, occurs at a lookback time of $\sim 8-10$ Gyr. Those analogues also show stellar mass-loss fraction $\gtrsim 10$ per cent. The lower panels of Fig. 4 show the evolution of the star formation rate (SFR). These systems fluctuate around a low average SFR at high redshift. In most cases, their SFR is not notably affected by infall or even the first pericentric passage, such that the peak stellar mass is reached after infall. Instead, star formation ends suddenly after two to three pericentres (3-6 Gyr) in response to an extremely rapid and neartotal loss of gas from the system at that time (seen in the cyan lines in the upper panels). This behaviour is common to the majority of the Fornax analogues.

The evolution of Fornax analogues after infall contrasts strongly with that of Sculptor and Leo I analogues, four examples of which are shown in Fig. 5. These systems have less dramatic differences between peak mass and current mass, and their infall times are typically more recent ( $\sim-9$ Gyr ago), consistent with previous studies of the likely infall times of different MW dSphs (Rocha et al. 2013). Their gas content and SFR fall to zero more quickly after infall, around the first apocentre unless their orbit stays far from the Galactic Centre the whole time (e.g. SL4).

The differences seen in the post-infall evolution of gas mass and the effects on the star formation histories of different analogues are interesting, and reminiscent of well-established differences in the stellar populations of observed dSphs (notably the presence of intermediate-age stars in Fornax). The catastrophic removal of gas is likely to be the result of ram-pressure stripping (Gunn \& Gott 1972).

Comparing different panels in Fig. 4, we also find that the level of tidal stripping is correlated with orbital properties, such as pericentre distance, number of pericentre passages and infall time. 

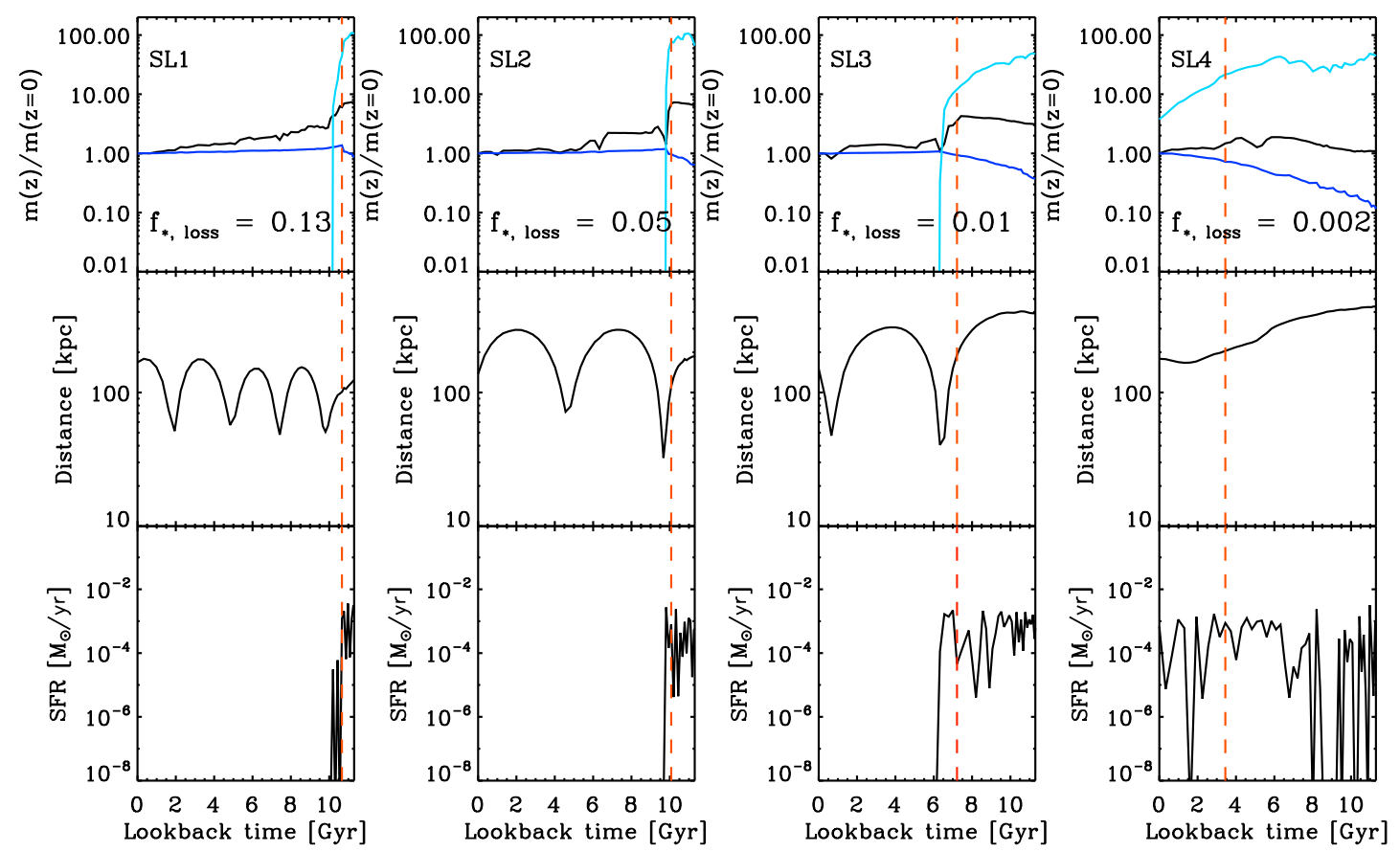

Figure 5. The same as Fig. 4, except here for four Sculptor and Leo I analogues (SL1-SL4).

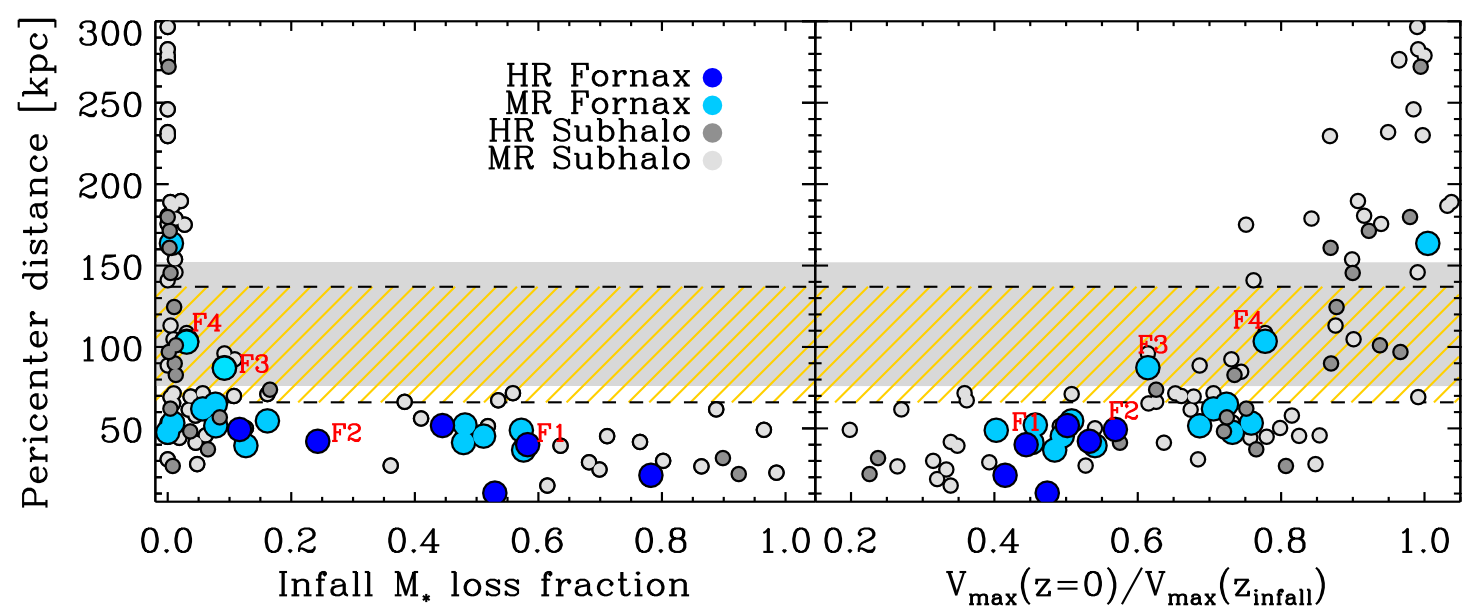

Figure 6. Pericentre distance as a function of stellar mass-loss fraction (left-hand panel) and $V_{\max }(z=0) / V_{\max }\left(z_{\text {infall }}\right)$ (right-hand panel) for subhaloes with stellar mass in the range $6 \times 10^{6} \leq M_{*} \leq 6 \times 10^{7} \mathrm{M}_{\odot}$ at $z=0$, which is the luminosity range of our Fornax analogues. The colour scheme of the points is the same as Fig. 2. The yellow shaded areas mark the 95 per cent confidence interval range of orbital perigalacticon from Piatek et al. (2007), and the grey shaded areas are derived perigalacticon 95 per cent confidence interval range with Galactic potentials derived from APOSTLE simulations. The same observed Fornax velocity information (see Piatek et al. 2007) is adopted in all perigalacticon calculations here (see Section 5.3 for details).

\subsection{Orbital motions}

We now compare the orbital motions of our analogue subhaloes with the observed motions of real dSphs, and thus determine whether the tidal forces on the simulated galaxies are representative of those experienced by real dSphs.

Fornax, Sculptor and Leo I all have measured proper motions from which their orbits can be reconstructed. These orbits are, however, subject to assumptions about the shape and amplitude of the Galactic potential. For instance, using measured radial velocities and proper motions of stars in Fornax, Piatek et al. (2007) estimate its perigalacticon, $r_{\mathrm{p}}$, and apogalacticon, $r_{\mathrm{a}}$, adopting a Galactic potential model from Johnston, Spergel \& Hernquist (1995). They find 95 percent confidence intervals of $66<r_{\mathrm{p}}<137 \mathrm{kpc}$ and $142<r_{\mathrm{a}}<242 \mathrm{kpc}$. The implied eccentricity of the orbit, $e=\left(r_{\mathrm{a}}-r_{\mathrm{p}}\right) /\left(r_{\mathrm{a}}+r_{\mathrm{p}}\right)$, is then in the range $0.11<e<0.38$.

In Fig. 6, we show the pericentre distance as a function of stellar mass-loss fraction (left-hand panel) and $V_{\max }(z=0) / V_{\max }\left(z_{\text {infall }}\right)$ (right-hand panel) for subhaloes with stellar mass in the range $6 \times 10^{6} \leq M_{\star} \leq 6 \times 10^{7} \mathrm{M}_{\odot}$, which is the luminosity range of our Fornax analogues. The yellow shaded areas mark the 95 percent confidence interval range of orbital perigalacticon from Piatek et al. (2007) (with their assumed MW potential), and the grey shaded areas are the 95 per cent confidence intervals for perigalacticon derived using the same orbital velocity measurements in combination with the potentials taken directly from the APOSTLE simulations.

To account for the response of the Fornax motion in the APOSTLE MW potentials, we derive the range of latest orbital 
Table 1. Pericentre distances $\left(r_{\mathrm{p}}\right)$ of Fornax derived with different assumptions regarding the Galactic potential and the current distance of Fornax. 'Ap-1', 'Ap-4' and 'Ap-11' refer to different HR APOSTLE simulations (see Section 2 for details). 'G1' and 'G2' indicate the two different MW-like galaxies in each simulation. Here we show pericentre predictions with two different assumptions for the current Fornax distance: $D=138$ or $147 \mathrm{kpc}$.

\begin{tabular}{lccccc}
\hline Galactic potential & $\begin{array}{c}M_{200} \\
\left(10^{12} \mathrm{M}_{\odot}\right)\end{array}$ & $\begin{array}{c}r_{\mathrm{p}}(D=138 \mathrm{kpc}) \\
(\mathrm{kpc})\end{array}$ & $\begin{array}{c}95 \text { per cent conf. } \\
(\mathrm{kpc})\end{array}$ & $\begin{array}{c}r_{\mathrm{p}}(D=147 \mathrm{kpc}) \\
(\mathrm{kpc})\end{array}$ & $\begin{array}{c}95 \text { per cent conf. } \\
(\mathrm{kpc})\end{array}$ \\
\hline AP-1 G1 & 1.66 & 128 & $(76,149)$ & 140.0 & $(93,165)$ \\
AP-1 G2 & 1.10 & 135 & $(113,152)$ & 144.0 & $(121,167)$ \\
AP-4 G1 & 1.38 & 133 & $(97,151)$ & 144.0 & $(113,167)$ \\
AP-4 G2 & 1.35 & 134 & $(102,151)$ & 144.0 & $(116,167)$ \\
AP-11 G1 & 0.99 & 135 & $(111,152)$ & 145.0 & $(121,167)$ \\
AP-11 G2 & 0.80 & 136 & $(114,152)$ & 145.0 & $(123,167)$ \\
Johnston et al.(1995) & - & 125 & $(74,148)$ & 138.0 & $(90,164)$ \\
\hline
\end{tabular}

pericentre distance using the equations that describe motion of a bound star in a spherical potential (Binney \& Tremaine 2008) with the observed Fornax radial motion and proper motion from Piatek et al. (2007). We assume that the potential is static. The pericentre and apocentre distances are then obtained by solving for $r$ in the following equation:

$\frac{1}{r^{2}}+\frac{2(E-\phi(r))}{L^{2}}=0$

where $E$ is the specific energy of the orbit, $L$ is the specific angular momentum and $\phi(r)$ is the potential. We have verified that this procedure, assuming the spherically averaged simulated potential at $z=0$, recovers accurately the last orbital pericentre and apocentre distances of the actual orbits of the subhaloes in the same simulations. We compute the 95 per cent confidence interval on the pericentre distance by Monte Carlo sampling from the error distributions of Fornax distance, radial velocity and proper motion, assuming these distributions to be Gaussian.

We find that the derived orbital pericentre and apocentre ranges vary with the assumption of the underlying Galactic potential. We have considered the six HR Galactic potentials from APOSTLE and the Galactic potential model from Johnston et al. (1995). We list the results of these calculations in Table 1.

We find that a population of Fornax analogues has a pericentre distance smaller than the lower bound on that of Fornax itself, more so for the analogues experiencing the greatest stellar tidal stripping. For the analogues that do lie within the shaded regions in Fig. 6 (both from the MR simulations), the highest stellar mass-loss fraction is $\lesssim 10$ per cent, which corresponds to $\sim 6.7 \times 10^{5} \mathrm{M}_{\odot}$ of tidally stripped stars. By interpolating along the approximate locus of the analogues in Fig. 6, we can infer that the observed bounds on the likely pericentre of Fornax are consistent with stellar mass-loss fractions of up to $\lesssim 15-20$ per cent.

We note that even though a majority of the Fornax analogues are heavily tidally stripped, most of them have orbits that are not consistent with the orbital properties (e.g. pericentre distance) inferred from the measurements of the motion of Fornax. Precisely matching the real orbital properties of Fornax is difficult due to the small sample size in our simulations. As a result, only two subhaloes satisfy all constraints when the orbital data are included. In addition to the implications for the formation of Fornax, this may have interesting implications for the MW potential. For example, in Table 1, we show that a more massive Galactic potential predicts smaller pericentre distances. If the MW halo mass is less than $\sim 10^{12} \mathrm{M}_{\odot}$, the 95 per cent confidence lower bound on the pericentre is $\sim 110 \mathrm{kpc}$ (see Table 1). This is also inconsistent with any of our Fornax analogues.
There are several studies presenting proper motion values for Fornax using different techniques (e.g. Dinescu et al. 2004; Walker, Mateo \& Olszewski 2008; Méndez et al. 2011). Those results usually have much larger error than the value in Piatek et al. (2007), and the quoted mean proper motion values roughly consistent with each other within $1 \sigma$ error except the results from Méndez et al. (2011) (see fig. 7 in their paper). We also test our orbital pericentre and apocentre calculation with these proper motion measurement, for example, from Walker et al. (2008). We find that the large uncertainties of these measurement result in poor constraints on the derived orbital properties. The possible pericentre distance range is $\sim 20-160 \mathrm{pc}$, assuming the current Fornax distance is $147 \mathrm{kpc}$ away, which covers significantly a much wider range than the results using Piatek et al. (2007) values. We therefore adopted the value from Piatek et al. (2007), which has the best precision among the existing measurement, in our Fornax orbital property study here.

In the case of Sculptor, a similar orbital study was carried out by Piatek et al. (2006). They obtain 95 percent confidence intervals on perigalacticon and apogalacticon of $31<r_{\mathrm{p}}<83 \mathrm{kpc}$ and $97<r_{\mathrm{a}}<313 \mathrm{kpc}$, implying $0.26<e<0.60$ with the Galactic potential model from Johnston et al. (1995). In the case of Leo I, a study of Hubble Space Telescope proper motion measurements was carried out by Sohn et al. (2013). Using three different mass models for the Galactic potential with total virial masses of $1.0 \times 10^{12}$, $1.5 \times 10^{12}$ and $2.0 \times 10^{12} \mathrm{M}_{\odot}$ respectively, they infer a perigalacticon occurring $1.05 \pm 0.09 \mathrm{Gyr}$ ago at a galactocentric distance of $r_{\mathrm{p}}=91 \pm 36 \mathrm{kpc}$. On this basis, they estimate that Leo I entered the MW virial radius $2.33 \pm 0.21 \mathrm{Gyr}$ ago and is most likely on its first infall. Most of our Sculptor/Leo I analogues lie within those perigalacticon and apogalacticon ranges, although some of them have higher orbital eccentricities.

\section{TIDAL DEBRIS}

In this section, we study the surface brightness of the outer regions of the analogues, including their tidal debris, in order to assess the detectability of tidal features. We also investigate the possibility that tidally stripped star may contaminate kinematic observations of real dSphs.

\subsection{Do dSphs have stellar tidal tails?}

In Fig. 7, we show the time evolution of the stellar mass surface density distribution for three HR dSph analogues: F1, F2 and SL2. In the leftmost panels, which correspond to these galaxies at their times of infall, the star particles are deeply embedded within their 

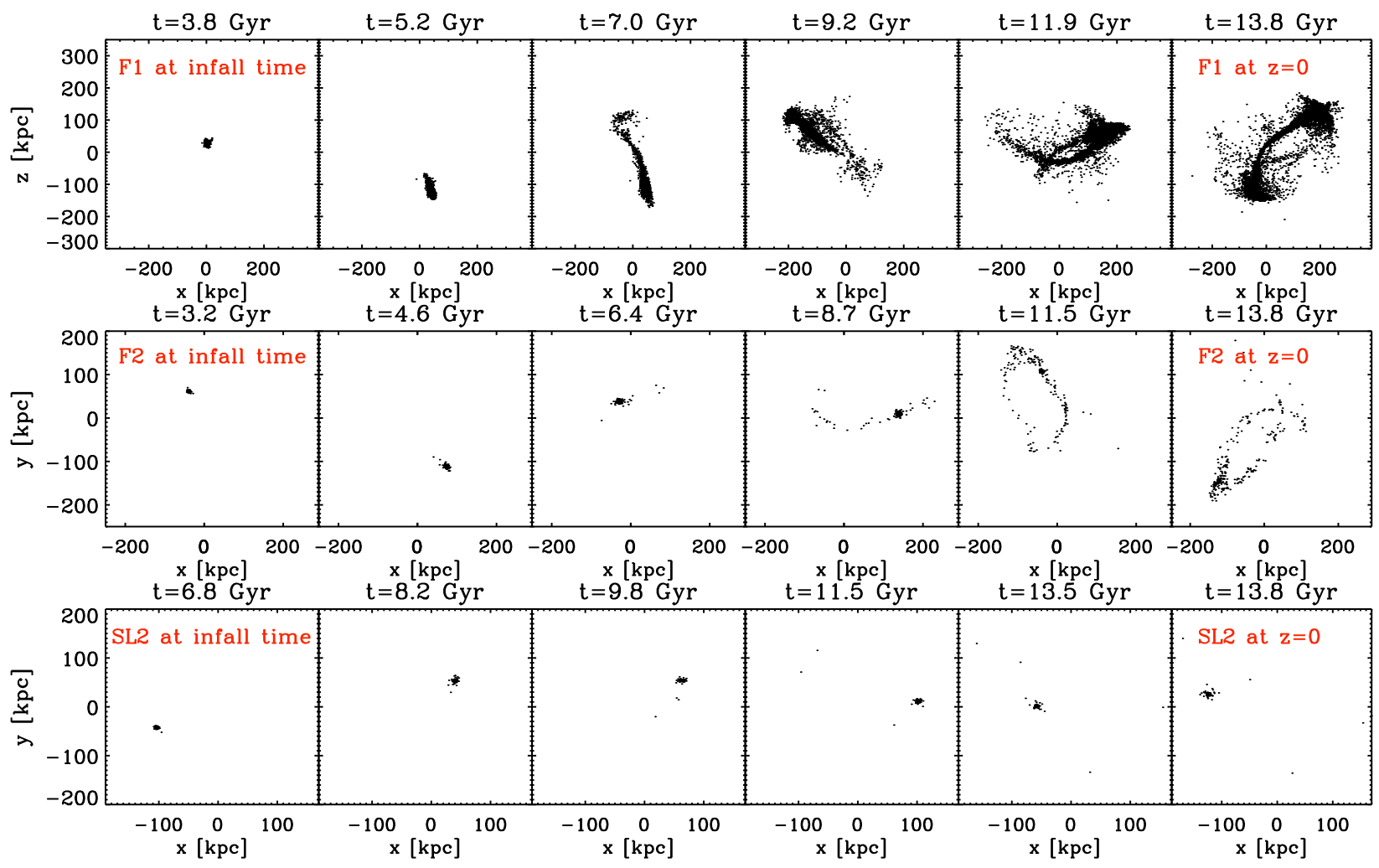

Figure 7. Projected spatial distribution of the star particles from two Fornax analogues (upper two rows, F1 and F2) and one Sculptor/Leo I analogue (lower row, SL2) as a function of the age of the Universe. These images include all star particles that are gravitationally bound to those dSphs at their infall time or at any time thereafter. For both Fornax analogues, tidally stripped star particles can be seen to extend far beyond the main bodies of the galaxies. For the Sculptor/Leo I analogues, there are no obvious tidal features, only a very small number of stripped star particles.

host haloes, as expected. As time evolves (from the left- to righthand side), we see that tidal stripping of stars from F1 and F2 leads to the development of leading and trailing streams that approximately trace their obits. Our results in Section 5.3 imply that Fornax may have lost up to 10-20 per cent of its infall stellar mass (equivalent to a stellar mass $\sim 7 \times 10^{5} \mathrm{M}_{\odot}$ ). Among the HR analogues, ${ }^{3} \mathrm{~F} 2$ is closest to having this amount of mass-loss. It is therefore likely to be a much better predictor of tidal features that may be observed around Fornax than F1, which has lost $f_{\star}$, loss $\sim 58$ per cent of its stars. F2 has $f_{\star}$, loss $\sim 24$ per cent, which is about $\sim 6.3 \times 10^{5} \mathrm{M}_{\odot}$ in stellar mass, but this is nevertheless sufficient to generate extended coherent tidal features. In contrast, SL2 does not have clear tidal tails, with only a handful of unbound star particles $\left(f_{\star}\right.$, loss $\sim 5$ per cent $)$. Indeed, as is shown in Fig. 1, most of the Sculptor and Leo I analogues exhibit very little stellar mass-loss, usually less than 20 percent. The qualitative features of SL2 shown in Fig. 7 are representative of such analogues.

The upper panels of Fig. 8 illustrate the star particle distribution of F2 at different phases along its orbit: in the upper panel, at the observed distance of Fornax $(D=140 \mathrm{kpc})$, and in the lower panels, at each perigalacticon. F2 has three perigalactic passages after its infall $\sim 10$ Gyr ago. Even after the first perigalacticon, the galaxy exhibits clear signs of tidal stripping.

Perhaps the most obvious reason that the majority of Fornax analogues show tidal features resulting from stellar stripping, whereas most of the Sculptor/Leo I analogues do not, is simply because the Fornax analogues must have lost more mass overall in order

${ }^{3}$ The resolution of the MR analogues is too low to study faint tidal features. to be identified as such. Fig. 2 demonstrates that stellar stripping correlates with total mass-loss. Similar finding is illustrated in fig. 8 in Sawala et al. (2016), where Fornax is shown to have very high stellar-to-total mass ratio and will likely be explained by tidal stripping. Another factor is the extent of the stellar distribution at the time of infall. In Fig. 9, we show the DM and stellar density profiles of F2 and SL2 at their perigalacticons and at $z=0$. At infall, their host subhaloes have similar halo masses, $\sim 4 \times 10^{9} \mathrm{M}_{\odot}$. At the second perigalactic passages $(t=9.5 \mathrm{Gyr}$ for $\mathrm{F} 2, t=13.1 \mathrm{Gyr}$ for SL2), their DM distributions (for the mass that SUBFIND considers bound) are both truncated at $r \sim 10 \mathrm{kpc}$. Figs 7 and 8 clearly show that while F2 already exhibits extended stream-like features at this second perigalacticon, SL2 has not lost significant stellar mass. This difference arises because, at infall, the stellar distribution in F2 extends up to $r \sim 10 \mathrm{kpc}$, whereas stars in SL2 are relatively more deeply embedded, extending only to $r \leq 7 \mathrm{kpc}$. As the subhaloes lose weakly bound DM to tides after infall, the subhalo tidal radius of F2 becomes comparable to the extent of its stellar distribution. The greater compactness of stars in SL2 at infall explains its apparently greater resilience to stellar stripping, despite the overall similarity in the halo mass and total mass evolution of the two galaxies.

\subsection{Surface densities of stellar tidal features}

We now provide estimates of the (typically very low) surface brightness of the tidal features seen in the simulations and compare these to known tidal features. We again focus on Fornax analogue F2 at $z=0$, with a galactocentric distance of $207 \mathrm{kpc}$, to provide an upper bound on the surface brightness estimate. 

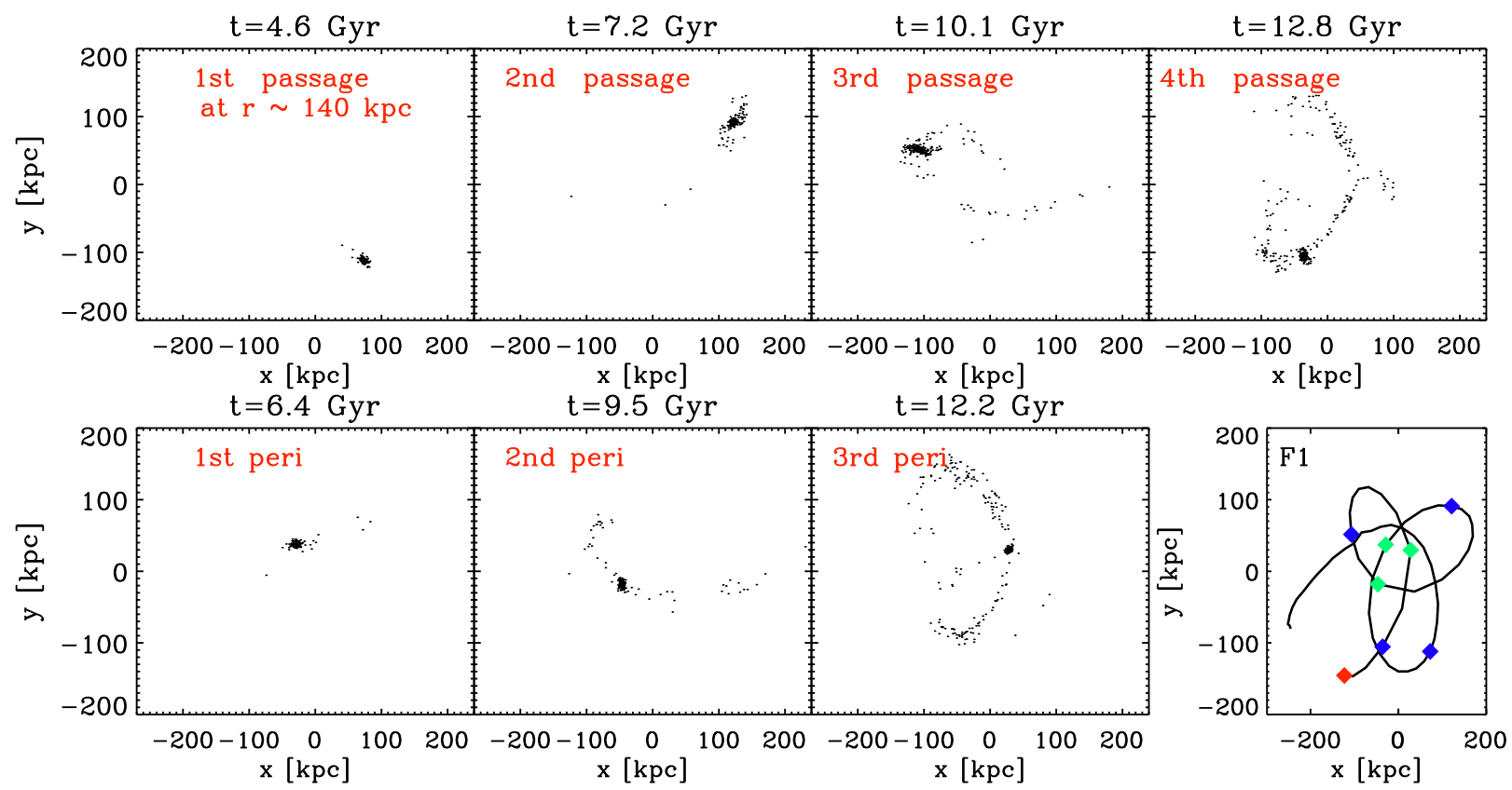

Figure 8. Projected spatial distribution of all star particles bound to F2 at infall or any time thereafter, at different times $t$. The upper panels show the star particle distribution when the galaxy is at a galactocentric distance $\sim 140 \mathrm{kpc}$ (the current distance of Fornax). The lower panels show equivalent distributions when F2 is at each of its perigalactic passages. The orbit of F2 is shown in the lower right-hand panel. Blue points along the orbit mark the locations with a galactocentric distance of $140 \mathrm{kpc}$ and green points mark perigalacticons. The red diamond marks the present-day location of the satellite.

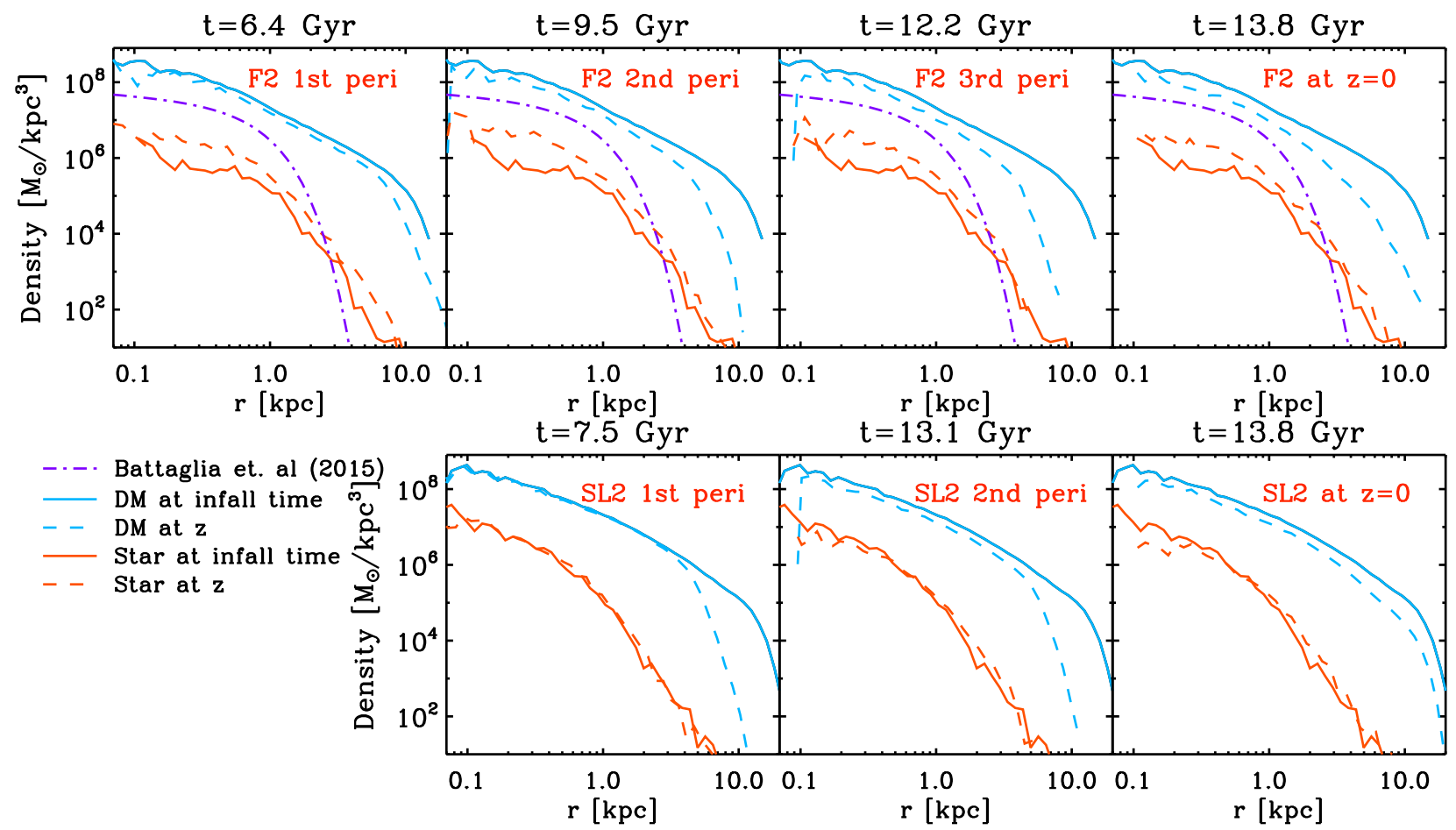

Figure 9. DM (blue lines) and stellar (red lines) density profiles of one Fornax analogue (F2, upper panels) and one Sculptor analogue (SL2, lower panels) at successive perigalacticons (from the left- to right-hand side) and at $z=0$ (rightmost panels). The profiles include only the gravitationally bound particles in each system. The solid lines denote the profiles at infall $(z \sim 2.2$ for F2 and $z \sim 0.86$ for SL2), and the dashed lines denote the profiles at later times. For F2, we also show the initial stellar density profile from Battaglia et al. (2015) as a purple dash-dotted line. The DM profiles for both F2 and SL1 continuously become shallower and lose mass in the outer region due to tidal disruption. The stellar distribution of F2 is more extended than that of SL1. For F2, the stellar densities increase at the centre after infall because star formation continues down to $z=0.4$, as shown in Fig. 4 . 

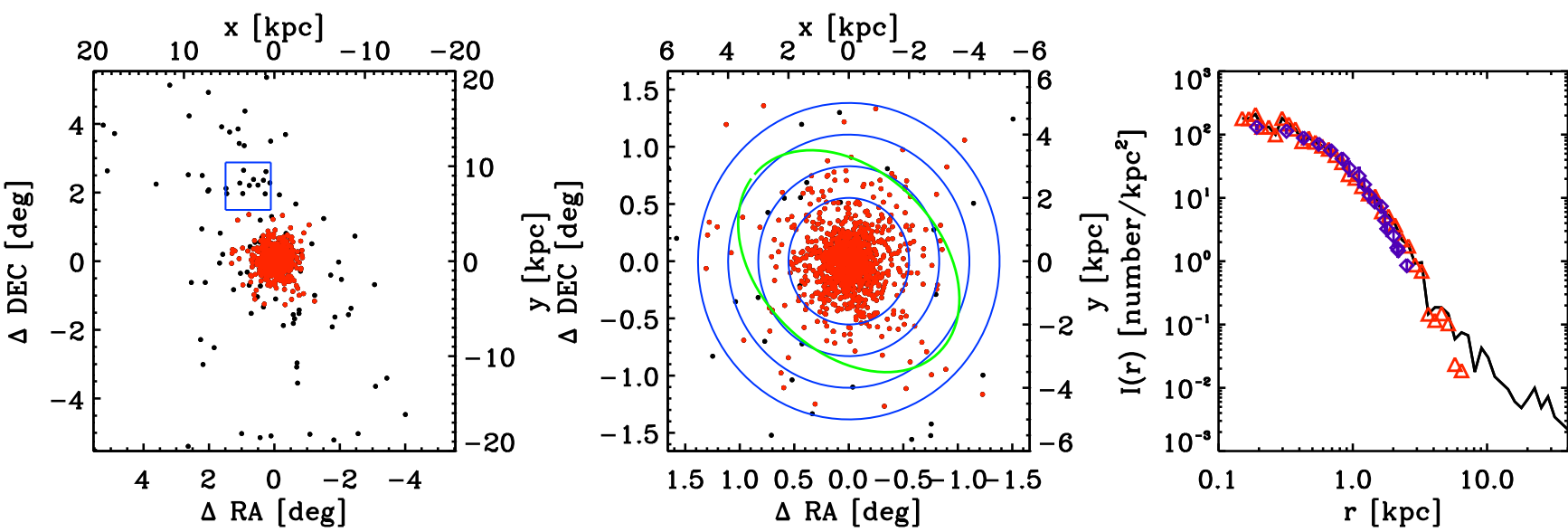

Figure 10. Spatial distribution of star particles in F2 (projected along the LOS to the centre of the main halo) at $z=0$. Red points/symbols mark the distribution of currently bound stars, and black points/lines the stripped stars. Left-hand panel: the particle distribution within a box $40 \mathrm{kpc}$ on a side $\left(\sim 10^{\circ}\right.$ for $\left.D=204 \mathrm{kpc}\right)$. The average surface brightness within the blue square ( $5 \mathrm{kpc}$ on a side) is $\sim \Sigma \sim 32.6 \mathrm{mag} \mathrm{arcsec}^{-2}$. Middle panel: a zoom-in view of the galaxy F2 (12 kpc on a side). We draw annuli (blue circles) around the galaxy with radii $2-5 \mathrm{kpc}\left(\sim 0.8^{\circ}-2.0^{\circ}\right)$ and $1-\mathrm{kpc}$ spacing to estimate surface brightness (see the text) around the stellar 'tidal radius' of Fornax (i.e. the apparent extent of the stellar distribution, $r_{\mathrm{t}}=69.1 \operatorname{arcmin}$ (Battaglia et al. 2006), shown as a black dashed ellipse). Right-hand panel: projected stellar number density profile for F2. The black line shows the total density of bound and stripped stars together. The red triangles mark the density for bound stars only. The purple diamonds (with $1 \sigma$ error) show the observed stellar surface density of Fornax from Coleman \& de Jong (2008).

We obtain a simple measure of surface brightness by drawing a few regions of interest around the simulated dwarf galaxies and along their stellar tidal tails. We use all the star particles lying within these regions, assuming a stellar mass-to-light ratio $M_{\star} / L=1$, to calculate an average apparent surface brightness. For the purpose of this exercise, we choose a fiducial projection along radial direction between the subhalo and galactic halo centre.

In Fig. 10, we show the spatial distributions of gravitationally bound star particles in F2 (red points) and star particles that were previously bound to F2 but have been unbound by tides (black points) at $z=0$. In the left-hand panel, we show all the star particles lying within a $40 \times 40 \mathrm{kpc}^{2}$ box projected along the $z$-axis of the simulation coordinate system. We can clearly see the tidally stripped stars extending along the $y$-direction. Within a $5 \times 5 \mathrm{kpc}^{2}$ box about $2^{\circ}$ away from the galaxy, the surface brightness of an overdense region is $\Sigma \sim 32.6 \mathrm{mag} \operatorname{arcsec}^{-2}$. The enclosed stellar mass within this region is $M_{\star} \sim 7.0 \times 10^{4} \mathrm{M}_{\odot}$. For comparison, the surface brightness of the Virgo overdensity discovered by SDSS is $\Sigma_{r} \sim 32.5 \mathrm{mag} \operatorname{arcsec}^{-2}$ (Jurić, Ivezić \& Brooks 2008), and the surface brightness of the Sagittarius dwarf Northern stream is $\Sigma_{V} \sim 31 \mathrm{mag} \operatorname{arcsec}^{-2}$ (Martínez-Delgado et al. 2004). The stellar overdensity in Eridanus-Phoenix recently discovered by DES has $\Sigma_{r} \sim 32.8 \mathrm{mag} \mathrm{arcsec}^{-2}$ (Li et al. 2016). These magnitudes are comparable to the surface brightness of the tidal feature from F2; therefore, similar features could be detected in ongoing or existing imaging surveys.

We also examine the stellar structure at the edge of the F2 galaxy. As shown in the middle panel of Fig. 10, we draw four annuli centred on the galaxy with a radius of $2-5 \mathrm{kpc}(\sim 0.55-1.4)$ and $1-\mathrm{kpc}$ spacing. Using naive visual inspection, there is no obvious stellar tidal structure or elongation of the body of the galaxy within $5 \mathrm{kpc}$, although this may be simply because the resolution of the simulation is not sufficient to capture any faint structures. The average surface brightness of the annulii are $\Sigma \sim 31.23$ (2-3 kpc), 32.8(3-4 kpc) and $33.6 \mathrm{mag} \operatorname{arcsec}^{-2}(4-5 \mathrm{kpc})$. The surface brightness rapidly drops below the reach of current surveys beyond $r>3-4 \mathrm{kpc}$ ( $r>50.5-67.4 \operatorname{arcmin}$ for $D=204 \mathrm{kpc}$, or $r>73.7-98.2 \operatorname{arcmin}$ for $D=140 \mathrm{kpc}$ ). This is consistent with the stellar 'tidal radius' of a King-model fit to the light profile of Fornax, $r_{\mathrm{t}}=69.1 \mathrm{arcmin}$ (Battaglia et al. 2006), beyond which the stellar distribution is indistinguishable from the background.

We have also demonstrated in the right-hand panel of Fig. 10 that the shape of F2's light profile agrees well with observations of Fornax itself (blue diamond points; Coleman et al. 2005). Although there are stars gravitationally bound to F2 at $r>3-4 \mathrm{kpc}$, their density would be indistinguishable from the background, at least with current imaging surveys. We show the surface number density of bound (red) and unbound (black) star particles in the right-hand panel of Fig. 10. The projected stellar density profile in the inner region of F2 is well resolved and its shape agrees well with that of Fornax overall (we have rescaled the amplitude of the simulated profile to match approximately that of the observed data, which is based on counts of giant stars). There is a steeper drop at $r>1.2 \mathrm{kpc}$ in the observed profile. The bound star particles in F2 extend up to $r \sim 9 \mathrm{kpc}$ and continue as a diffuse tidally stripped stellar tail.

We note that stellar tidal features are usually identified in surveys by 'matched filtering', using colour and magnitude cuts based on theoretical or empirical model isochrones to isolate, for example, main-sequence turn-of or red giant branch (RGB) stars from the background (Rockosi et al. 2002; Belokurov et al. 2006). Here we do not implement such a procedure, assuming instead that all stars in the tidal features are detectable. Our numbers therefore serve as upper limits on surface brightness. Due to the limited particle resolution, we can only report average surface brightness estimations over large areas because the fine structures of tidal features are not resolved in our simulations. In reality, if there are any overdense structures around MW dSphs, they could contain smaller scale fluctuations brighter than our estimates. We nevertheless find that there are several features along our simulated dSph tidal streams that have surface densities comparable to known stellar overdensities. The presence of such structures around galaxies such as Fornax can therefore be constrained in the near future by wide-field imagining observations. 

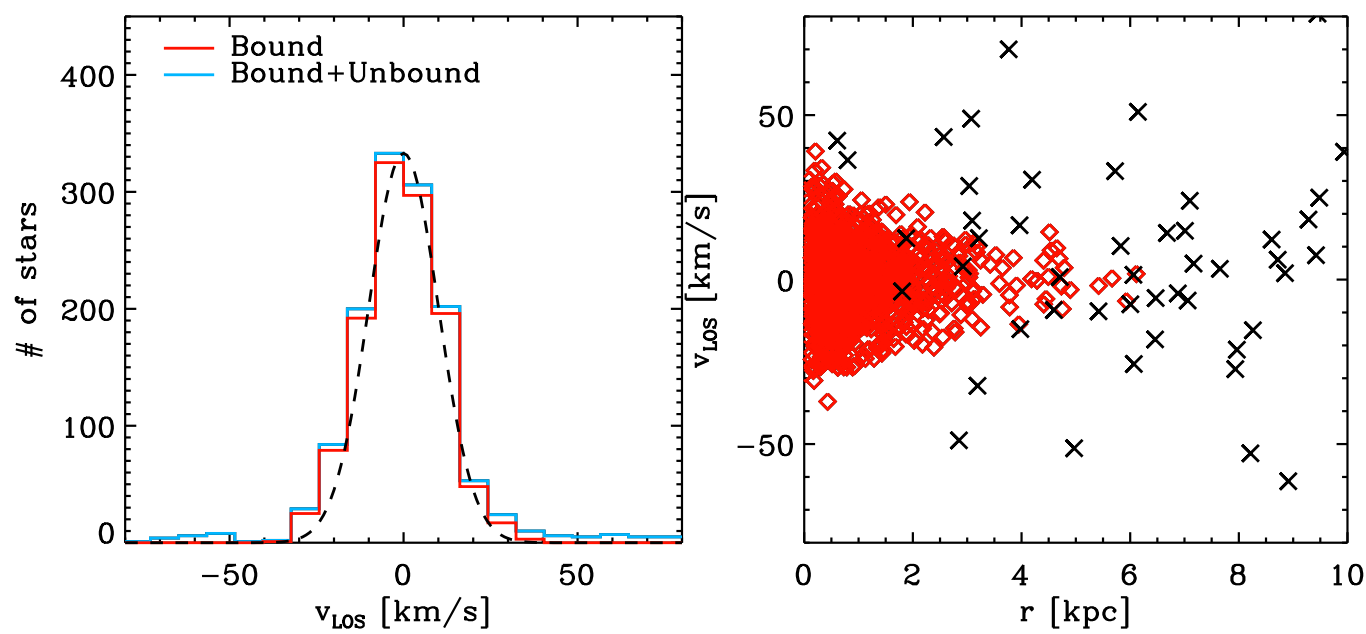

Figure 11. LOS velocity distribution of stars (velocity relative to the subhalo motion) for the F2 subhalo. The LOS direction is along the radial vector from the Galactic Centre to F2. Left-hand panel: histograms of stars that are currently gravitationally bound to F2 (red) and those that have ever been bound to F2, at infall or any time thereafter (blue). A Gaussian distribution with width $10 \mathrm{~km} \mathrm{~s}^{-1}$ is shown as a black dashed line. Right-hand panel: LOS velocity distribution as a function of radius. Red diamonds are bound stars and black crosses are stripped stars.

\subsection{Stellar kinematic contaminations from tidal debris}

In systems with very high mass-to-light ratios, such as the MW $\mathrm{dSphs}$, stellar kinematics provide a direct probe of the distribution of DM on very small scales. Such measurements are now one of the principle tests of the validity of the CDM model. However, the samples of stars used to study the kinematics of MW dSphs can be contaminated by tidally stripped stars that are not in equilibrium with the system. This contamination will affect the precision of dynamical mass estimates based on stellar velocities (Klimentowski et al. 2007). Here we demonstrate that gravitationally unbound stars are indeed likely to be confused with bound member stars in conventional stellar kinematic observations. We also demonstrate that depending on the projected orientation of tidal tails with respect to the LOS, these unbound stars will bias stellar velocity dispersion measurements.

In the left-hand panels of Fig. 11, we show the LOS velocity distribution of stars (red: bound only, blue: both bound and unbound) in the F2 subhalo, projected along the radial direction (i.e. along the vector from the centre of the galactic halo to the centre of F2) with velocity measured relative to the peculiar velocity of F2. The LOS velocity distributions are close to Gaussian, centred at zero relative velocity with average dispersion $\sim 10 \mathrm{~km} \mathrm{~s}^{-1}$ (black dashed curves). In the right-hand panel, we plot the velocity distribution as a function of radius for both the bound (red diamonds) and stripped stars (black crosses). Some of the stripped stars have velocities much larger than those of bound stars at the same radius. However, a good fraction of these lie within the range of the velocity distribution of bound stars, between -40 and $40 \mathrm{~km} \mathrm{~s}^{-1}$.

Fig. 11 indicates that a simple, non-iterative, cut-off in velocity, as commonly applied to kinematic samples (e.g. Walker et al. 2006), may be subject to kinematic contamination from tidally stripped stars. There are other methods that can be combined to determine membership probabilities for stars that utilize information such as the spatial distribution, the colour-magnitude diagram (CMD), the metallicity of stars and the full velocity distribution (e.g. Simon \& Geha 2007; Walker et al. 2009). However, since stripped stars were associated with the target galaxies in the past, their metallicities and CMDs are likely to be similar to those of the stars that remain bound. The spatial distribution may be more informative because stars closer to the centres of galaxies are more likely to be bound, although this method can only provide relative probabilities. Stripped stars in the foreground or background may still contaminate the velocity sample at small projected radii (see the right-hand panel of Fig. 11).

In Fig. 12, we show that the unbound stars have characteristic velocity signatures. They can inflate the stellar velocity dispersion and change the stellar velocity anisotropy to an extent that depends on the orientation of the tidal stream and the peculiar velocity of the galaxy along the LOS direction. Similar results have been obtained by several previous studies (e.g. Read et al. 2006; Klimentowski et al. 2007). For instance, Klimentowski et al. (2007) studied $\mathrm{N}$-body simulations of a dwarf galaxy on a highly eccentric orbit. They found that when the dwarf is observed along its tidal tails, kinematic samples are most strongly contaminated by unbound stars from the tails (e.g. figs 5 and 6 in their paper). In the upper panels of Fig. 12, we plot the velocity dispersion for F2 along the LOS (blue points) and in a direction perpendicular to the LOS (orange points). We only include stars with LOS velocities $\pm 40 \mathrm{~km} \mathrm{~s}^{-1}$ relative to the peculiar velocity of F2, as these would be identified as possible member stars using the width of the velocity distribution alone. We find the velocity dispersion is inflated by unbound stars at $r>3 \mathrm{kpc}$ by a factor of $>30$ per cent (depending on the LOS projection) relative to the velocity dispersion of bound stars at $r \sim 3 \mathrm{kpc}$.

In the lower panels of Fig. 12, we show the velocity dispersion anisotropy $\beta(r)$, which denotes the ratio between the tangential and radial components of the velocity dispersion. At a given radius,

$\beta(r)=1-\frac{\sigma_{\mathrm{t}}^{2}}{2 \sigma_{r}^{2}}$,

where $\sigma_{\mathrm{t}}^{2}$ is the tangential component of the velocity dispersion and $\sigma_{r}^{2}$ is the radial component. In F2, the velocity anisotropy for the bound stars (lower right-hand panel) is consistent with zero at most of the radii, with a variation between -0.1 and 0.5 . This indicates that isotropic orbits are good approximations for bound stars in the F2 galaxy. However, at $r>2 \mathrm{kpc}$, where unbound stars start to dominate, the velocity anisotropy can deviate significantly from zero. For example, the curve in lower left-hand panel shows an increase in anisotropy. This result can be explained by the large radial motions of stripped stars along the stellar stream. 


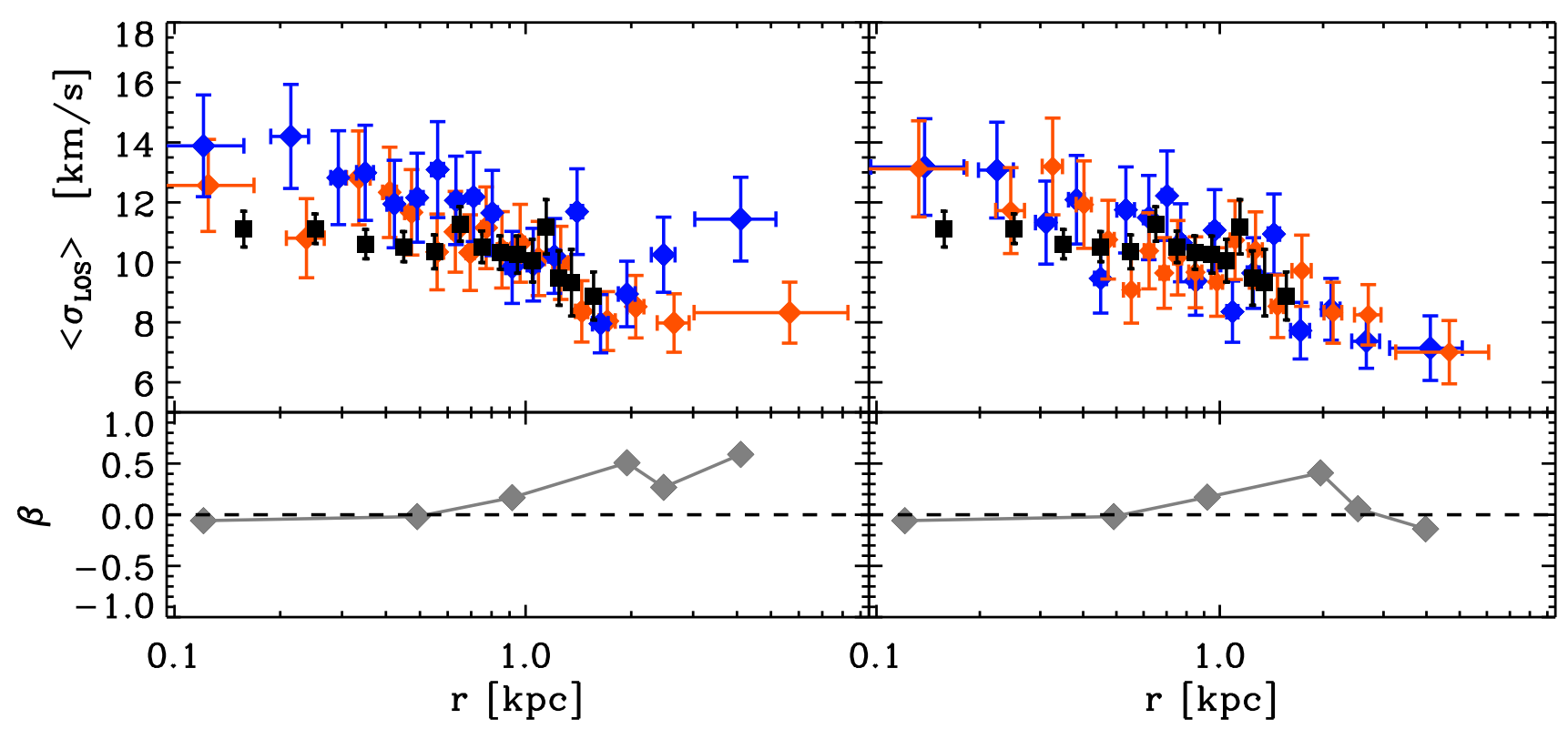

Figure 12. LOS velocity dispersion (upper panels) and velocity anisotropy (lower panels) of stars in Fornax analogue F2. All the stars are selected to have LOS velocities in the range $\pm 40 \mathrm{~km} \mathrm{~s}^{-1}$ relative to the LOS velocity of F2 as a whole. The right-hand panels show results for stars that are currently gravitationally bound to F2, while the left-hand panels show results of all stars that have ever been bound to F2 at any time since its infall. We plot the LOS velocity projected along the radius vector from the Galactic Centre to F2 (blue points) and, for comparison, along a direction perpendicular to that vector (orange points).

\subsection{Comparison to previous work on Fornax tidal evolution}

Battaglia et al. (2015) construct a set of $N$-body simulations to study the effect of tides on Fornax. However, in contrast to our results, they do not find any evidence for stellar tidal features. To understand this discrepancy, we compare the initial conditions of their simulations with our Fornax analogues. They adopted two orbital set-ups: one with perigalactic radius $r_{\mathrm{p}}=118 \mathrm{kpc}$ and apogalactic radius $r_{\mathrm{a}}=152 \mathrm{kpc}$, yielding an orbital eccentricity $e=0.13$, and another with $e=0.4$ and $r_{\mathrm{p}}=61 \mathrm{kpc}$. Since the orbit of our subhalo F2 is quite similar to their second orbital configuration (first $r_{\mathrm{p}} \sim 68 \mathrm{kpc}$ and $r_{\mathrm{a}} \sim 153 \mathrm{kpc}$ after infall), we can conclude that the difference is not entirely due to the orbits.

The initial DM mass adopted in Battaglia et al. (2015) was $3 \times 10^{9} \mathrm{M}_{\odot}$, which is similar to the infall mass of $\mathrm{F} 2$, i.e. $4 \times 10^{9} \mathrm{M}_{\odot}$. The infall stellar mass in our simulation is lower than their initial stellar mass $\left(5 \times 10^{7} \mathrm{M}_{\odot}\right.$ versus our value of $3 \times$ $\left.10^{6} \mathrm{M}_{\odot}\right)$. In addition, in order to increase the possibility of generating stellar tidal disruption, Battaglia et al. (2015) assumed a cored DM density profile.

Therefore, purely on the basis of its subhalo mass and DM density profile, our Fornax DM subhalo should be more resilient to tidal forces than their subhalo. However, when we compare the stellar density profiles, which are shown in Fig. 9, we see that their stellar distribution is much more concentrated than ours. In the upper panels of Fig. 9, the purple dash-dotted lines show the stellar density profile of Battaglia et al. (2015). It declines very rapidly around Fornax's nominal King stellar 'tidal radius' of $\sim 3 \mathrm{kpc}$. In contrast, our profiles have an extended distribution up to $\sim 7 \mathrm{kpc}$ (at $z=0$ ). However, as shown in Section 6.2, the surface brightness of F2 drops quickly at $r>3 \mathrm{kpc}$, at which point it falls below the limiting background density of current observations. It is therefore quite likely that, in reality, the Fornax stellar distribution extends far beyond the apparent 'tidal radius'. This may explain why our conclusions regarding tidal features around Fornax differ from those of Battaglia et al. (2015).

\section{CONCLUSION AND DISCUSSION}

We have used the HR APOSTLE hydrodynamical simulations to investigate, for the first time, the stellar tidal stripping of classical MW dSphs in the range $M_{\star}=10^{6}-10^{8} \mathrm{M}_{\odot}$ in a cosmological context. This cosmological context is important because the factors that determine the severity of tidal effects, including the assembly history and structure of the potential well of the host galaxy, the mass and structure of the satellite galaxies, and their infall times, stellar content and orbits, are all self-consistently taken into account. In these simulations, we find many examples of stellar stripping of satellites due to tidal interactions with their host; $\sim 30$ per cent of satellites lose more than 20 percent of their stellar mass between their time of infall and the present day. This fraction is remarkably high given that only a handful of MW dSphs display clear signs of tidal tails or strong structural distortions that could be attributed to tidal stirring. However, given the difficulty of detecting diffuse lowsurface brightness tidal features, the apparent contradictions can be explained by a lack of sufficiently deep wide-area photometric data. Ongoing and future deep imaging surveys, such as DES and LSST, will be able to verify this in the near future.

We have identified Fornax analogues that best match the observed orbital properties of Fornax. We note that many of our Fornax analogue subhaloes are severely tidally stripped, but they tend to have lower orbital pericentre distance than the predictions derived from measurements of the velocity of Fornax. This puzzling tension may indicate that Fornax's orbit is in some way unusual, or provide constraints on the Galactic potential. We leave this work to a future study with more detailed modelling of the Galactic potential and orbital simulations of Fornax.

Nevertheless, those Fornax analogues that match the orbital properties have typically lost $\sim 10-20$ per cent of their infall stellar mass and exhibit faint stellar tidal streams along their orbit. This tidal debris is generally low in surface brightness, but with overdense regions that are comparable to currently known stellar overdensities and streams in the MW. We also show that these Fornax analogues 
may have diffuse but nevertheless bound stellar components extending beyond the conventional Fornax stellar 'tidal radius'. These extended envelopes are difficult to distinguish from background stars.

Our results are interesting in the context of recent observational and theoretical studies of Fornax. For example, Bate et al. (2015) have used VST ATLAS survey data to show that a population of Fornax RGB stars extends all the way out to the Fornax stellar 'tidal radius' defined by Battaglia et al. (2006). This observation is in line with the predictions of our simulation, and differs from the conclusions of Battaglia et al. (2015) that stars in Fornax do not extend beyond that radius, and that Fornax has undergone no significant stellar tidal stripping. The lack of significant stellar tidal features in our Sculptor and Leo I analogues can, however, be explained by their compact stellar distribution, even though their DM tidal radius (different than the stellar one) may be similar to that of the Fornax analogues after a few pericentric passages.

Having identified Fornax, Sculptor and Leo I analogues in the simulations on the basis of their observed stellar kinematics and luminosities, we find that they exhibit different mass assembly histories and star formation histories. Fornax analogues in particular suffer a significant mass-loss, with early infall $\sim 8-10$ Gyr ago. They exhibit continuous star formation activity after infall, although this activity has generally ceased by the present day. On the other hand, the mass-loss of Sculptor and Leo I analogues is much less severe. These analogues tend to have more recent infall times than Fornax, with star formation suppressed very rapidly after infall.

As noted by Wang et al. (2016), the correlation between infall time and star formation time-scale could also be useful in testing the nature of DM because different DM models predict different infall times. As an example, in the case of Fornax, CDM models always predict infall at lookback times of $\sim 8-9$ Gyr ago, whereas alternatives, such as WDM or DDM models, predict infall times as recent as 3-4 Gyr ago. It is interesting to note that Fornax does show signs of recent star formation activity (Weisz et al. 2015) $\sim 2$ Gyr ago. This short time-scale for shutting down star formation may be in tension with infall $>5$ Gyr ago. Thus, it is important to understand how the star formation time-scales of dSphs can be used to constrain their likely orbital history, which, in turn, may enable stronger tests of the nature of DM.

Finally, we have discussed the potential for kinematic contamination by tidally stripped stars in the context of dSph stellar velocity dispersion measurements. We have shown that unbound stars can inflate the stellar velocity dispersion and change the average stellar velocity anisotropy in the outskirts of $\mathrm{dSphs}$. We find that a conventional cut on heliocentric velocities cannot exclude all the unbound particles, nor can metallicity cuts or selections based on CMD filtering, since the contaminating stars were once gravitationally bound member stars. On the other hand, these kinematic features can also serve as a signature to detect tidal debris.

\section{ACKNOWLEDGEMENTS}

We would like to thank Nitya Kallivayalil, Manoj Kaplinghat, Mike Boylan-Kolchin, Robyn Sanderson, Josh Simon and Ting Li for useful discussions. MYW and LES acknowledge support from National Science Foundation grant PHY-1522717. APC is supported by a COFUND/Durham Junior Research Fellowship under EU grant 267209 and by STFC (ST/L00075X/1). TS acknowledges the support of the Academy of Finland grant 1274931. JFN was supported by a Visiting Professorship Grant from the Leverhulme Trust VP1-2014-021. This work used the DiRAC Data Centric sys- tem at Durham University, operated by the Institute for Computational Cosmology on behalf of the STFC DiRAC HPC Facility (www.dirac.ac.uk). This equipment was funded by BIS National E-infrastructure capital grant ST/K00042X/1, STFC capital grants ST/H008519/1 and ST/K00087X/1, STFC DiRAC Operations grant ST/K003267/1 and Durham University. DiRAC is part of the National E-Infrastructure.

\section{REFERENCES}

Barber C., Starkenburg E., Navarro J. F., McConnachie A. W., 2015, MNRAS, 447, 1112

Bate N. F., McMonigal B., Lewis G. F., Irwin M. J., Gonzalez-Solares E., Shanks T., Metcalfe N., 2015, MNRAS, 453, 690

Battaglia G. et al., 2006, A\&A, 459, 423

Battaglia G., Helmi A., Tolstoy E., Irwin M., Hill V., Jablonka P., 2008, ApJ, 681, L13

Battaglia G., Irwin M., Tolstoy E., de Boer T., Mateo M., 2012, ApJ, 761, L31

Battaglia G., Sollima A., Nipoti C., 2015, MNRAS, 454, 2401

Belokurov V. et al., 2006, ApJ, 642, L137

Binney J., Tremaine S., 2008, Galactic Dynamics, 2nd edn. Princeton Univ. Press, Princeton, NJ

Boylan-Kolchin M., Bullock J. S., Kaplinghat M., 2011, MNRAS, 415, L40 Boylan-Kolchin M., Bullock J. S., Kaplinghat M., 2012, MNRAS, 422, 1203

Choi P. I., Guhathakurta P., Johnston K. V., 2002, AJ, 124, 310

Coleman M. G., de Jong J. T. A., 2008, ApJ, 685, 933

Coleman M. G., Da Costa G. S., Bland-Hawthorn J., Freeman K. C., 2005 , AJ, 129, 1443

Cooper A. et al., 2010, MNRAS, 406, 744

Crain R. A. et al., 2015, MNRAS, 450, 1937

Crnojević D. et al., 2014, MNRAS, 445, 3862

de Boer T. J. L. et al., 2012, A\&A, 544, A73

Dinescu D. I., Keeney B. A., Majewski S. R., Girard T. M., 2004, AJ, 128 , 687

Dooley G. A., Peter A. H. G., Vogelsberger M., Zavala J., Frebel A., 2016, MNRAS, 461, 710

Errani R., Peñarrubia J., Tormen G., 2015, MNRAS, 449, L46

Fattahi A. et al., 2016a, MNRAS, 457, 844

Fattahi A., Navarro J. F., Sawala T., Frenk C. S., Sales L. V., Oman K., Schaller M., Wang J., 2016b, MNRAS, preprint (arXiv:1607.06479)

Furlong M. et al., 2015, MNRAS, 450, 4486

Geha M., Guhathakurta P., Rich R. M., Cooper M. C., 2006, AJ, 131, 332

Gunn J. E., Gott J. R., III, 1972, ApJ, 176, 1

Guo Q., Cooper A. P., Frenk C., Helly J., Hellwing W. A., 2015, MNRAS, 454,550

Ibata R. A., Gilmore G., Irwin M. J., 1994, Nature, 370, 194

Johnston K. V., Spergel D. N., Hernquist L., 1995, ApJ, 451, 598

Jurić M. et al., 2008, ApJ, 673, 864

Klimentowski J., Łokas E. L., Kazantzidis S., Prada F., Mayer L., Mamon G. A., 2007, MNRAS, 378, 353

Koch A., Wilkinson M. I., Kleyna J. T., Gilmore G. F., Grebel E. K., Mackey

A. D., Evans N. W., Wyse R. F. G., 2007, ApJ, 657, 241

Komatsu E. et al., 2011, ApJS, 192, 18

Li T. S. et al., 2016, ApJ, 817, 135

Li Y.-S., De Lucia G., Helmi A., 2010, MNRAS, 401, 2036

Lovell M. R., Frenk C. S., Eke V. R., Jenkins A., Gao L., Theuns T., 2014, MNRAS, 439, 300

McConnachie A. W., 2012, AJ, 144, 4

McMonigal B. et al., 2014, MNRAS, 444, 3139

Martínez-Delgado D., Gómez-Flechoso M. Á., Aparicio A., Carrera R., 2004, ApJ, 601, 242

Mateo M. L., 1998, ARA\&A, 36, 435

Mateo M., Olszewski E. W., Walker M. G., 2008, ApJ, 675, 201

Mayer L., Governato F., Colpi M., Moore B., Quinn T., Wadsley J., Stadel J., Lake G., 2001, ApJ, 547, L123 
Méndez R. A., Costa E., Gallart C., Pedreros M. H., Moyano M., Altmann M., 2011, AJ, 142, 93

Muñoz R. R. et al., 2006, ApJ, 649, 201

Muñoz R. R., Majewski S. R., Johnston K. V., 2008, ApJ, 679, 346

Peñarrubia J., Navarro J. F., McConnachie A. W., 2008, ApJ, 673, 226

Piatek S., Pryor C., Bristow P., Olszewski E. W., Harris H. C., Mateo M., Minniti D., Tinney C. G., 2006, AJ, 131, 1445

Piatek S., Pryor C., Bristow P., Olszewski E. W., Harris H. C., Mateo M., Minniti D., Tinney C. G., 2007, AJ, 133, 818

Power C., Navarro J. F., Jenkins A., Frenk C. S., White S. D. M., Springel V., Stadel J., Quinn T., 2003, MNRAS, 338, 14

Read J. I., Wilkinson M. I., Evans N. W., Gilmore G., Kleyna J. T., 2006, MNRAS, 367, 387

Rocha M., Peter A. H. G., Bullock J. S., Kaplinghat M., Garrison-Kimmel S., Oñorbe J., Moustakas L. A., 2013, MNRAS, 430, 81

Rockosi C. M. et al., 2002, AJ, 124, 349

Roderick T. A., Jerjen H., Mackey A. D., Da Costa G. S., 2015, ApJ, 804, 134

Sawala T. et al., 2016, MNRAS, 457, 1931

Schaye J. et al., 2015, MNRAS, 446, 521

Simon J. D., Geha M., 2007, ApJ, 670, 313

Smolčić V., Zucker D. B., Bell E. F., Coleman M. G., Rix H. W., Schinnerer E., Ivezić Ž., Kniazev A., 2007, AJ, 134, 1901

Sohn S. T., Besla G., van der Marel R. P., Boylan-Kolchin M., Majewski S. R., Bullock J. S., 2013, ApJ, 768, 139
Springel V., 2005, MNRAS, 364, 1105

Springel V., White S. D. M., Tormen G., Kauffmann G., 2001, MNRAS, 328,726

Strigari L. E., Frenk C. S., White S. D. M., 2010, MNRAS, 408, 2364

Tomozeiu M., Mayer L., Quinn T., 2016, ApJ, 818, 193

Trayford J. W. et al., 2015, MNRAS, 452, 2879

Walker M., 2013, in Oswalt T. D., Gilmore G., eds, Planets, Stars and Stellar Systems Vol. 5. Springer Science+Business Media, Dordrecht, p. 1039

Walker M. G., Mateo M., Olszewski E. W., Bernstein R., Wang X., Woodroofe M., 2006, AJ, 131, 2114

Walker M. G., Mateo M., Olszewski E. W., 2008, ApJ, 688, L75

Walker M. G., Mateo M., Olszewski E. W., Sen B., Woodroofe M., 2009, AJ, 137, 3109

Walker M. G., Mateo M., Olszewski E. W., 2009, AJ, 137, 3100

Wang M.-Y., Peter A. H. G., Strigari L. E., Zentner A. R., Arant B., GarrisonKimmel S., Rocha M., 2014, MNRAS, 445, 614

Wang M.-Y., Strigari L. E., Lovell M. R., Frenk C. S., Zentner A. R., 2016, MNRAS, 457, 4248

Weisz D. R., Dolphin A. E., Skillman E. D., Holtzman J., Gilbert K. M., Dalcanton J. J., Williams B. F., 2015, ApJ, 804, 136

Wolf J., Martinez G. D., Bullock J. S., Kaplinghat M., Geha M., Muñoz R. R., Simon J. D., Avedo F. F., 2010, MNRAS, 406, 1220

This paper has been typeset from a $\mathrm{T}_{\mathrm{E}} \mathrm{X} / \mathrm{L} \mathrm{T}_{\mathrm{E}} \mathrm{X}$ file prepared by the author. 